Geometry $8 \mathcal{T}$ Topology

Volume 5 (2001) 369-398

Published: 20 April 2001

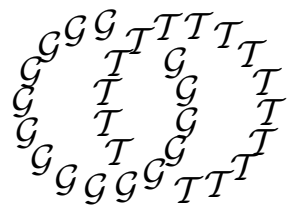

\title{
The size of triangulations supporting a given link
}

\author{
SimON A King \\ Institut de Recherche Mathématique Avancée \\ Strasbourg, France \\ Email: king@math.u-strasbg.fr
}

\begin{abstract}
Let $\mathcal{T}$ be a triangulation of $S^{3}$ containing a link $L$ in its 1 -skeleton. We give an explicit lower bound for the number of tetrahedra of $\mathcal{T}$ in terms of the bridge number of $L$. Our proof is based on the theory of almost normal surfaces.
\end{abstract}

AMS Classification numbers Primary: 57M25, 57Q15

Secondary: 68Q25

Keywords: Link, triangulation, bridge number, Rubinstein-Thompson algorithm, normal surfaces

Proposed: Walter Neumann

Seconded: Cameron Gordon, David Gabai

(C) Geometry $\&$ Topology Publications
Received: 12 September 2000

Accepted: 8 April 2001 


\section{Introduction}

In this paper, we prove the following result.

Theorem 1 Let $L \subset S^{3}$ be a tame link with bridge number $b(L)$. Let $\mathcal{T}$ be a triangulation of $S^{3}$ with $n$ tetrahedra such that $L$ is contained in the 1 -skeleton of $\mathcal{T}$. Then

$$
n>\frac{1}{14} \sqrt{\log _{2} b(L)},
$$

or equivalently

$$
b(L)<2^{196 n^{2}}
$$

The definition of the bridge number can be found, for instance, in [2]. So far as is known to the author, Theorem 1 gives the first estimate for $n$ in terms of $L$ that does not rely on additional geometric or combinatorial assumptions on $\mathcal{T}$. We show in [13] that the bound for $b(L)$ in Theorem 1 can not be replaced by a sub-exponential bound in $n$. More precisely, there is a constant $c \in \mathbb{R}$ such that for any $i \in \mathbb{N}$ there is a triangulation $\mathcal{T}_{i}$ of $S^{3}$ with $\leq c \cdot i$ tetrahedra, containing a two-component link $L_{i}$ in its 1-skeleton with $b\left(L_{i}\right)>2^{i-2}$.

The relationship of geometric and combinatorial properties of a triangulation of $S^{3}$ with the knots in its 1 -skeleton has been studied earlier, see [6], [15], [1], [3], [7]. For any knot $K \subset S^{3}$ there is a triangulation of $S^{3}$ such that $K$ is formed by three edges, see [4]. Let $\mathcal{T}$ be a triangulation of $S^{3}$ with $n$ tetrahedra and let $K \subset S^{3}$ be a knot formed by a path of $k$ edges. If $\mathcal{T}$ is shellable (see [3]) or the dual cellular decomposition is shellable (see [1]), then $b(K) \leq \frac{1}{2} k$. If $\mathcal{T}$ is vertex decomposable then $b(K) \leq \frac{1}{3} k$, see [3].

We reduce Theorem 1 to Theorem 2 below, for which we need some definitions. Denote $I=[0,1]$. Let $M$ be a closed 3-manifold with a triangulation $\mathcal{T}$. The $i$-skeleton of $\mathcal{T}$ is denoted by $\mathcal{T}^{i}$. Let $S$ be a surface and let $H: S \times I \rightarrow M$ be an embedding, so that $\mathcal{T}^{1} \subset H\left(S^{2} \times I\right)$. A point $x \in \mathcal{T}^{1}$ is a critical point of $H$ if $H_{\xi}=H(S \times \xi)$ is not transversal to $\mathcal{T}^{1}$ in $x$, for some $\xi \in I$. We call $H$ a $\mathcal{T}^{1}$-Morse embedding, if $H$ is in general position with respect to $\mathcal{T}^{1}$; we give a more precise definition in Section 5 . Denote by $c\left(H, \mathcal{T}^{1}\right)$ the number of critical points of $H$.

Theorem 2 Let $\mathcal{T}$ be a triangulation of $S^{3}$ with $n$ tetrahedra. There is a $\mathcal{T}^{1}$-Morse embedding $H: S^{2} \times I \rightarrow S^{3}$ such that $\mathcal{T}^{1} \subset H\left(S^{2} \times I\right)$ and $c\left(H, \mathcal{T}^{1}\right)<2^{196 n^{2}}$. 
For a link $L \subset \mathcal{T}^{1}$, it is easy to see that $b(L) \leq \frac{1}{2} \min _{H}\left\{c\left(H, \mathcal{T}^{1}\right)\right\}$, where the minimum is taken over all $\mathcal{T}^{1}$-Morse embeddings $H: S^{2} \times I \rightarrow S^{3}$ with $L \subset H\left(S^{2} \times I\right)$. Thus Theorem 1 is a corollary of Theorem 2 .

Our proof of Theorem 2 is based on the theory of almost 2-normal surfaces. Kneser [14] introduced 1-normal surfaces in his study of connected sums of 3 -manifolds. The theory of 1 -normal surfaces was further developed by Haken (see [8], [9]). It led to a classification algorithm for knots and for sufficiently large 3-manifolds, see for instance [11], [17]. The more general notion of almost 2-normal surfaces is due to Rubinstein [19]. With this concept, Rubinstein and Thompson found a recognition algorithm for $S^{3}$, see [19], [22], [16]. Based on the results discussed in a preliminary version of this paper [12], the author [13] and Mijatović [18] independently obtained a recognition algorithm for $S^{3}$ using local transformations of triangulations.

We outline here the proof of Theorem 2. Let $\mathcal{T}$ be a triangulation of $S^{3}$ with $n$ tetrahedra. If $S \subset S^{3}$ is an embedded surface and $S \cap \mathcal{T}^{1}$ is finite, then set $\|S\|=\operatorname{card}\left(S \cap \mathcal{T}^{1}\right)$. Let $S_{1}, \ldots, S_{k} \subset S^{3}$ be surfaces. A surface that is obtained by joining $S_{1}, \ldots, S_{k}$ with some small tubes in $M \backslash \mathcal{T}^{1}$ is called a tube sum of $S_{1}, \ldots, S_{k}$.

Based on the Rubinstein-Thompson algorithm, we construct a system $\tilde{\Sigma} \subset S^{3}$ of pairwise disjoint $2-$ normal $2-$ spheres such that $\|\tilde{\Sigma}\|$ is bounded in terms of $n$ and any 1 -normal sphere in $S^{3} \backslash \tilde{\Sigma}$ is parallel to a connected component of $\tilde{\Sigma}$. The bound for $\|\tilde{\Sigma}\|$ can be seen as part of a complexity analysis for the Rubinstein-Thompson algorithm and relies on results on integer programming.

A $\mathcal{T}^{1}$-Morse embedding $H$ then is constructed "piecewise" in the connected components of $S^{3} \backslash \tilde{\Sigma}$, which means the following. There are numbers $0<\xi_{1}<$ $\cdots<\xi_{m}<1$ such that:

(1) $\left\|H_{0}\right\|=\left\|H_{1}\right\|=0$.

(2) There is one critical value of $H \mid\left[0, \xi_{1}\right]$, corresponding to a vertex $x_{0} \in \mathcal{T}^{0}$. The set of critical points of $H \mid\left[\xi_{m}, 1\right]$ is $\mathcal{T}^{0} \backslash\left\{x_{0}\right\}$.

(3) For any $i=1, \ldots, m$, the sphere $H_{\xi_{i}}$ is a tube sum of components of $\tilde{\Sigma}$.

(4) The critical points of $H \mid\left[\xi_{i}, \xi_{i+1}\right]$ are contained in a single connected component $N_{i}$ of $S^{3} \backslash \tilde{\Sigma}$.

(5) The function $\xi \mapsto\left\|H_{\xi}\right\|$ is monotone in any interval $\left[\xi_{i}, \xi_{i+1}\right]$, for $i=$ $1, \ldots, m-1$.

This is depicted in Figure 1, where the components of $\tilde{\Sigma}$ are dotted. The components $N_{i}$ run over all components of $S^{3} \backslash \tilde{\Sigma}$ that are not regular neighbourhoods of vertices of $\mathcal{T}$. Thus an estimate for $m$ is obtained by an estimate 


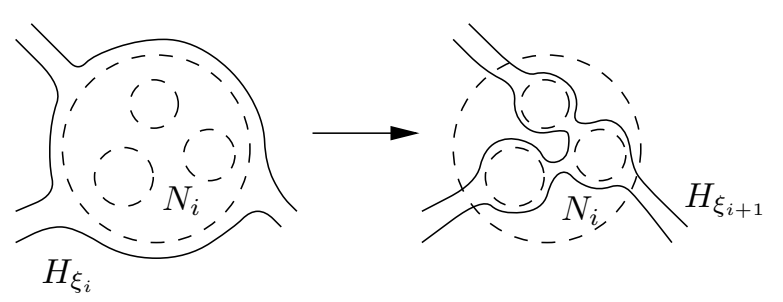

Figure 1: About the construction of $H$

for the number of components of $\tilde{\Sigma}$. By monotonicity of $\left\|H_{\xi}\right\|$, the number of critical points in $N_{i}$ is bounded by $\frac{1}{2}\left\|\partial N_{i}\right\| \leq \frac{1}{2}\|\tilde{\Sigma}\|$. This together with the bound for $m$ yields the claimed estimate for $c\left(H, \mathcal{T}^{1}\right)$.

The main difficulty in constructing $H$ is to assure property (5). For this, we introduce the notions of upper and lower reductions. If $S^{\prime}$ is an upper (resp. lower) reduction of a surfaces $S \subset S^{3}$, then $S$ is isotopic to $S^{\prime}$ such that $\|\cdot\|$ is monotonely non-increasing under the isotopy. Let $N$ be a connected component of $S^{3} \backslash \tilde{\Sigma}$ with $\partial N=S_{0} \cup S_{1} \cup \cdots \cup S_{k}$. We show that there is a tube sum $S$ of $S_{1}, \ldots, S_{k}$ such that either $S$ is a lower reduction of $S_{0}$, or $S_{0}$ is an upper reduction of $S$. Finally, if $H_{\xi_{i}}$ is a tube sum of $S_{0}$ with some surface $S^{\prime} \subset S^{3} \backslash N$, then $H \mid\left[\xi_{i}, \xi_{i+1}\right]$ is induced by the lower reductions (resp. the inverse of the upper reductions) relating $S_{0}$ with $S$. Then $H_{\xi_{i+1}}$ is a tube sum of $S$ with $S^{\prime}$, assuring properties (3)-(5).

The paper is organized as follows. In Section 2, we recall basic properties of $k$-normal surfaces. It is well known that the set of 1 -normal surfaces in a triangulated 3-manifold is additively generated by so-called fundamental surfaces. In Section 3, we generalize this to 2-normal surfaces contained in sub-manifolds of triangulated 3-manifolds. The system $\tilde{\Sigma}$ of 2 -normal spheres is constructed in Section 4, in the more general setting of closed orientable 3-manifolds. In Section 5 , we recall the notions of almost $k$-normal surfaces (see [16]) and of impermeable surfaces (see [22]), and introduce the new notion of split equivalence. We discuss the close relationship of almost 2-normal surfaces and impermeable surfaces. This relationship is well known (see [22], [16]), but the proofs are only partly available. For completeness we give a proof in the last Section 9. In Section 6 we exhibit some useful properties of almost 1 -normal surfaces. The notions of upper and lower reductions are introduced in Section 7. The proof of Theorem 2 is finished in Section 8.

In this paper, we denote by $\#(X)$ the number of connected components of a topological space $X$. If $X$ is a tame subset of a 3-manifold $M$, then $U(X) \subset M$ 
denotes a regular neighbourhood of $X$ in $M$. For a triangulation $\mathcal{T}$ of $M$, the number of its tetrahedra is denoted by $t(\mathcal{T})$.

Acknowledgements I would like to thank Professor Sergei V Matveev and my scientific supervisor Professor Vladimir G Turaev for many interesting discussions and for helpful comments on this paper.

\section{A survey of $k$-normal surfaces}

Let $M$ be a closed 3 -manifold with a triangulation $\mathcal{T}$. The number of its tetrahedra is denoted by $t(\mathcal{T})$. An isotopy $\bmod \mathcal{T}^{n}$ is an ambient isotopy of $M$ that fixes any simplex of $\mathcal{T}^{n}$ set-wise. Some authors call an isotopy $\bmod \mathcal{T}^{2}$ a normal isotopy.

Definition 1 Let $\sigma$ be a 2-simplex and let $\gamma \subset \sigma$ be a closed embedded arc with $\gamma \cap \partial \sigma=\partial \gamma$, disjoint to the vertices of $\sigma$. If $\gamma$ connects two different edges of $\sigma$ then $\gamma$ is called a normal arc. Otherwise, $\gamma$ is called a return.

We denote the number of connected components of a topological space $X$ by $\#(X)$. Let $\sigma$ be a $2-$ simplex with edges $e_{1}, e_{2}, e_{3}$. If $\Gamma \subset \sigma$ is a system of normal arcs, then $\Gamma$ is determined by $\Gamma \cap \partial \sigma$, up to isotopy constant on $\partial \sigma$, and $e_{1}$ is connected with $e_{2}$ by $\frac{1}{2}\left(\#\left(\Gamma \cap e_{1}\right)+\#\left(\Gamma \cap e_{2}\right)-\#\left(\Gamma \cap e_{3}\right)\right)$ arcs in $\Gamma$.

Definition 2 Let $S \subset M$ be a closed embedded surface transversal to $\mathcal{T}^{2}$. We call $S$ pre-normal, if $S \backslash \mathcal{T}^{2}$ is a disjoint union of discs and $S \cap \mathcal{T}^{2}$ is a union of normal arcs in the 2 -simplices of $\mathcal{T}$.

The set $S \cap \mathcal{T}^{1}$ determines the normal arcs of $S \cap \mathcal{T}^{2}$. For any tetrahedron $t$ of $\mathcal{T}$, the components of $S \cap t$, being discs, are determined by $S \cap \partial t$, up to isotopy fixed on $\partial t$. Thus we obtain the following lemma.

Lemma 1 A pre-normal surface $S \subset M$ is determined by $S \cap \mathcal{T}^{1}$, up to isotopy $\bmod \mathcal{T}^{2}$.

Definition 3 Let $S \subset M$ be a pre-normal surface and let $k$ be a natural number. If for any connected component $C$ of $S \backslash \mathcal{T}^{2}$ and any edge $e$ of $\mathcal{T}$ holds $\#(\partial C \cap e) \leq k$, then $S$ is $k$-normal. 

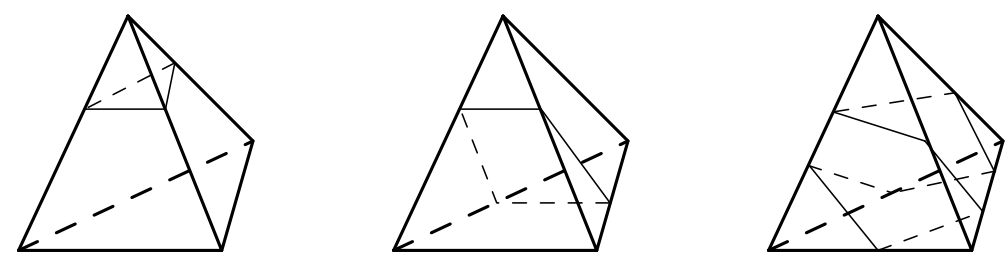

Figure 2: A triangle, a square and an octagon

We are mostly interested in $1-$ and $2-$ normal surfaces. Let $S$ be a $2-$ normal surface and let $t$ be a tetrahedron of $\mathcal{T}$. Then the components of $S \cap t$ are copies of triangles, squares and octagons, as in Figure 2. For any tetrahedron $t$, there are 10 possible types of components of $S \cap t$ : four triangles (one for each vertex of $t$ ), three squares (one for each pair of opposite edges of $t$ ), and three octagons. Thus there are $10 t(\mathcal{T})$ possible types of components of $S \backslash \mathcal{T}^{2}$. Up to isotopy mod $\mathcal{T}^{2}$, the set $S \backslash \mathcal{T}^{2}$ is described by the vector $\mathfrak{x}(S)$ of $10 t(\mathcal{T})$ non-negative integers that indicates the number of copies of the different types of discs occuring in $S \backslash \mathcal{T}^{2}$. Note that the 1 -normal surfaces are formed by triangles and squares only.

We will describe the non-negative integer vectors that represent $2-$ normal surfaces. Let $S \subset M$ be a 2 -normal surface and let $x_{t, 1}, \ldots, x_{t, 6}$ be the components of $\mathfrak{x}(S)$ that correspond to the squares and octagons in some tetrahedron $t$. It is impossible that in $S \cap t$ occur two different types of squares or octagons, since two different squares or octagons would yield a self-intersection of $S$. Thus all but at most one of $x_{t, 1}, \ldots, x_{t, 6}$ vanish for any $t$. This property of $\mathfrak{x}(S)$ is called compatibility condition.

Let $\gamma$ be a normal arc in a 2 -simplex $\sigma$ of $\mathcal{T}$ and $t_{1}, t_{2}$ be the two tetrahedra that meet at $\sigma$. In both $t_{1}$ and $t_{2}$ there are one triangle, one square and two octagons that contain a copy of $\gamma$ in its boundary. Moreover, each of them contains exactly one copy of $\gamma$. Let $x_{t_{i}, 1}, \ldots, x_{t_{i}, 4}$ be the components of $\mathfrak{x}(S)$ that correspond to these types of discs in $t_{i}$, where $i=1,2$. Since $\partial S=\emptyset$, the number of components of $S \cap t_{1}$ containing a copy of $\gamma$ equals the number of components of $S \cap t_{2}$ containing a copy of $\gamma$. That is to say $x_{t_{1}, 1}+\cdots+$ $x_{t_{1}, 4}=x_{t_{2}, 1}+\cdots+x_{t_{2}, 4}$. Thus $\mathfrak{x}(S)$ satisfies a system of linear Diophantine equations, with one equation for each type of normal arcs. This property of $\mathfrak{x}(S)$ is called matching condition. The next claim states that the compatibility and the matching conditions characterize the vectors that represent 2-normal surfaces. A proof can be found in [11], Chapter 9 . 
Proposition 1 Let $\mathfrak{x}$ be a vector of $10 t(\mathcal{T})$ non-negative integers that satisfies both the compatibility and the matching conditions. Then there is a 2-normal surface $S \subset M$ with $\mathfrak{x}(S)=\mathfrak{x}$.

Two 2-normal surfaces $S_{1}, S_{2}$ are called compatible if the vector $\mathfrak{x}\left(S_{1}\right)+\mathfrak{x}\left(S_{2}\right)$ satisfies the compatibility condition. It always satisfies the matching condition. Thus if $S_{1}$ and $S_{2}$ are compatible, then there is a 2 -normal surface $S$ with $\mathfrak{x}(S)=\mathfrak{x}\left(S_{1}\right)+\mathfrak{x}\left(S_{2}\right)$, and we denote $S=S_{1}+S_{2}$. Conversely, let $S$ be a 2-normal surface, and assume that there are non-negative integer vectors $\mathfrak{x}_{1}, \mathfrak{x}_{2}$ that both satisfy the matching condition, with $\mathfrak{x}(S)=\mathfrak{x}_{1}+\mathfrak{x}_{2}$. Then both $\mathfrak{x}_{1}$ and $\mathfrak{x}_{2}$ satisfy the compatibility condition. Thus there are 2-normal surfaces $S_{1}, S_{2}$ with $S=S_{1}+S_{2}$. The Euler characteristic is additive, i.e., $\chi\left(S_{1}+S_{2}\right)=\chi\left(S_{1}\right)+\chi\left(S_{2}\right)$, see [11].

Remark 1 The addition of 2-normal surfaces extends to an addition on the set of pre-normal surfaces as follows. If $S_{1}, S_{2} \subset M$ are pre-normal surfaces, then $S_{1}+S_{2}$ is the pre-normal surface that is determined by $\mathcal{T}^{1} \cap\left(S_{1} \cup S_{2}\right)$. The addition yields a semi-group structure on the set of pre-normal surfaces. This semi-group is isomorphic to the semi-group of integer points in a certain rational convex cone that is associated to $\mathcal{T}$. The Euler characteristic is not additive with respect to the addition of pre-normal surfaces.

\section{Fundamental surfaces}

We use the notations of the previous section. The power of the theory of $2-$ normal surfaces is based on the following two finiteness results.

Proposition 2 Let $S \subset M$ be a 2-normal surface comprising more than $10 t(\mathcal{T})$ two-sided connected components. Then two connected components of $S$ are isotopic $\bmod \mathcal{T}^{2}$.

This is proven in [9], Lemma 4, for 1-normal surfaces. The proof easily extends to 2 -normal surfaces.

Theorem 3 Let $N \subset M \backslash U\left(\mathcal{T}^{0}\right)$ be a sub-3-manifold whose boundary is a 1-normal surface. There is a system $F_{1}, \ldots, F_{q} \subset N$ of 2-normal surfaces such that

$$
\left\|F_{i}\right\|<\|\partial N\| \cdot 2^{18 t(\mathcal{T})}
$$

for $i=1, \ldots, q$, and any 2 -normal surface $F \subset N$ can be written as a sum $F=\sum_{i=1}^{q} k_{i} F_{i}$ with non-negative integers $k_{1}, \ldots, k_{q}$. 
The surfaces $F_{1}, \ldots, F_{q}$ are called fundamental. Theorem 3 is a generalization of a result of [10] that concerns the case $N=M \backslash U\left(\mathcal{T}^{0}\right)$.

The rest of this section is devoted to the proof of Theorem 3. The idea is to define a system of linear Diophantine equations (matching equations) whose non-negative solutions correspond to 2 -normal surfaces in $N$. The fundamental surfaces $F_{1}, \ldots, F_{q}$ correspond to the Hilbert base vectors of the equation system, and the bound for $\left\|F_{i}\right\|$ is a consequence of estimates for the norm of Hilbert base vectors. Note that in an earlier version of this paper [12], we proved Theorem 3 in essentially the same way, but using handle decompositions of 3-manifolds rather than triangulations.

Definition 4 A region of $N$ is a component $R$ of $N \cap$, for a closed tetrahedron $t$ of $\mathcal{T}$. If $\partial R \cap \partial N$ consists of two copies of one normal triangle or normal square then $R$ is a parallelity region.

Definition 5 The class of a normal triangle, square or octagon in $N$ is its equivalence class with respect to isotopies mod $\mathcal{T}^{2}$ with support in $U(N)$.

Let $t$ be a closed tetrahedron of $\mathcal{T}$, and let $R \subset t$ be a region of $N$. One verifies that if $R$ is not a parallelity region then $\partial R \cap \partial N$ either consists of four normal triangles ("type I") or of two normal triangles and one normal square ("type II"). If $R$ is of type I, then $R$ is isotopic $\bmod \mathcal{T}^{2}$ to $t \backslash U\left(\mathcal{T}^{0}\right)$, and any other region of $N$ in $t$ is a parallelity region. As in the previous section, $R$ contains four classes of normal triangles, three classes of normal squares and three classes of normal octagons. If $R$ is of type II, then $t$ contains at most one other region of $N$ that is not a parallelity region, that is then also of type II. A normal square or octagon in $t$ that is not isotopic $\bmod \mathcal{T}^{2}$ to a component of $\partial R \cap \partial N$ intersects $\partial R$. Thus $R$ contains two classes of normal triangles and one class of normal squares.

Let $m(N)$ be the number of classes of normal triangles, squares and octagons in regions of $N$ of types I and II. If $N$ has $k$ regions of type I, then $N$ has $\leq 2(t(\mathcal{T})-k)$ regions of type II, thus $m(N) \leq 10 k+6(t(\mathcal{T})-k) \leq 10 t(\mathcal{T})$. Let $\bar{m}(N)$ be the number of parallelity regions of $N$. It is easy to see that $\bar{m}(N) \leq \frac{1}{2} \#\left(\partial N \backslash \mathcal{T}^{2}\right) \leq \frac{1}{6}\|\partial N\| \cdot t(\mathcal{T})$.

Any 2-normal surface $F \subset N$ is determined up to isotopy $\bmod \mathcal{T}^{2}$ with support in $U(N)$ by the vector $\overline{\mathfrak{x}}_{N}(F)$ of $m(N)+\bar{m}(N)$ non-negative integers that count the number of components of $F \backslash \mathcal{T}^{2}$ in each class of normal triangles, squares and octagons. Let $\gamma_{1}, \gamma_{2} \subset \mathcal{T}^{2}$ be normal arcs, and let $R_{1}, R_{2}$ be two regions of $N$ with $\gamma_{1} \subset \partial R_{1}$ and $\gamma_{2} \subset \partial R_{2}$. For $i=1,2$, let $x_{i, 1}, \ldots, x_{i, m_{i}}$ be the 
components of $\overline{\mathfrak{x}}_{N}(F)$ that correspond to classes of normal triangles, squares and octagons in $R_{i}$ that contain $\gamma_{i}$ in its boundary. If $x_{1,1}+\cdots+x_{1, m_{1}}=x_{2,1}+$ $\cdots+x_{2, m_{2}}$ then we say that $\overline{\mathfrak{x}}_{N}(F)$ satisfies the matching equation associated to $\left(\gamma_{1}, R_{1} ; \gamma_{2}, R_{2}\right)$.

For $i=1,2, R_{i}$ contains one class of normal triangles that contain a copy of $\gamma_{i}$ in its boundary. If $R_{i}$ is not a parallelity region, then $R_{i}$ contains one class of normal squares that contain a copy of $\gamma_{i}$ in its boundary. If $K_{i}$ is of type I, then $K_{i}$ additionally contains two classes of normal octagons containing a copy of $\gamma_{i}$ in its boundary. Thus if $R_{i}$ is a parallelity region then $m_{i}=1$, if it is of type I then $m_{i}=4$, and if it is of type II then $m_{i}=2$.

For any 2-normal surface $F \subset N$, let $\mathfrak{x}_{N}(F) \in \mathbb{Z}_{\geq 0}^{m(N)}$ be the vector that collects the components of $\overline{\mathfrak{x}}_{N}(F)$ corresponding to the classes of normal triangles, squares and octagons in regions of $N$ of types I and II. As in the previous section, the vector $\mathfrak{x}_{N}(F)$ (resp. $\overline{\mathfrak{x}}_{N}(F)$ ) satisfies a compatibility condition, i.e., for any region $R$ of $N$ vanish all but at most one components of $\mathfrak{x}_{N}(F)$ (resp. $\left.\overline{\mathfrak{x}}_{N}(F)\right)$ corresponding to classes of squares and octagons in $R$.

Lemma 2 Suppose that any component of $N$ contains a region that is not a parallelity region. There is a system of matching equations concerning only regions of $N$ of types $I$ and $I I$, such that a vector $\mathfrak{x} \in \mathbb{Z}_{>0}^{m(N)}$ satisfies these equations and the compatibility condition if and only if there is a 2-normal surface $F \subset N$ with $\mathfrak{x}_{N}(F)=\mathfrak{x}$. The surface $F$ is determined by $\mathfrak{x}_{N}(F)$, up to isotopy in $N \bmod \mathcal{T}^{2}$.

Proof Let $\gamma \subset N \cap \mathcal{T}^{2}$ be a normal arc. Let $R_{1}, R_{2}$ be the two regions of $N$ that contain $\gamma$. Let $F \subset N$ be a 2 -normal surface. Since $\partial F=\emptyset$, the number of components of $F \cap R_{1}$ containing $\gamma$ and the number of components of $F \cap R_{2}$ containing $\gamma$ coincide. Thus $\overline{\mathfrak{x}}_{N}(F)$ satisfies the matching equation associated to $\left(\gamma, R_{1} ; \gamma, R_{2}\right)$. We refer to these equations as $N$-matching equations. We will transform the system of $N$-matching equations by eliminating the components of $\overline{\mathfrak{x}}_{N}(F)$ that do not belong to $\mathfrak{x}_{N}(F)$.

Let $\gamma_{1}, \gamma_{2} \subset \mathcal{T}^{2}$ be normal arcs, and let $R_{1}, R_{2}$ be two different regions of $N$ with $\gamma_{1} \subset \partial R_{1}$ and $\gamma_{2} \subset \partial R_{2}$. Assume that $R_{1}$ is a parallelity region of $N$. Then $m_{1}=1$, thus the matching equation associated to $\left(\gamma_{1}, R_{1} ; \gamma_{2}, R_{2}\right)$ is of the form $x_{1,1}=x_{2,1}+\cdots+x_{2, m_{2}}$. Hence we can eliminate $x_{1,1}$ in the $N-$ matching equations. For any region $R_{3}$ of $N$ and any normal arc $\gamma_{3} \subset \partial R_{3}$, the elimination transforms the matching equation associated to $\left(\gamma_{1}, R_{1} ; \gamma_{3}, R_{3}\right)$ into the matching equation associated to $\left(\gamma_{2}, R_{2} ; \gamma_{3}, R_{3}\right)$. We iterate the elimination process. Since any component of $N$ contains a region that is not a 
parallelity region, we eventually transform the system of $N$-matching equations to a system $\mathfrak{A}$ of matching equations that concern only regions of $N$ of types I and II.

Let $\mathfrak{x} \in \mathbb{Z}_{\geq 0}^{m(N)}$ be a solution of $\mathfrak{A} \cdot \mathfrak{x}=0$. By the elimination process, there is a unique extension of $\mathfrak{x}$ to a solution $\overline{\mathfrak{x}}$ of the $N$-matching equations. If $\mathfrak{x}$ satisfies the compatibility condition then so does $\overline{\mathfrak{x}}$, since a parallelity region contains at most one class of normal squares. Now the lemma follows by Proposition 1, that is proven in [11].

Proof of Theorem 3 It is easy to verify that if $R$ is a parallelity region then there is only one class of $2-$ normal pieces in $R$. If a component $N_{1}$ of $N$ is a union of parallelity regions, then $N_{1}$ is a regular neighbourhood of a 1-normal surface $F_{1} \subset N_{1}$, that has a connected non-empty intersection with each region of $N_{1}$. Any pre-normal surface in $N_{1}$ is a multiple of $F_{1}$ (thus, is 1-normal), see [8]. We have $\left\|F_{1}\right\|=\frac{1}{2}\left\|\partial N_{1}\right\|$. Thus by now we can suppose that any component of $N$ contains a region that is not a parallelity region.

By Lemma 2, the $\mathfrak{x}$-vectors of 2-normal surfaces in $N$ satisfy a system of linear equations $\mathfrak{A} \cdot \mathfrak{x}=0$. By the following well known result on Integer Programming (see [21]), the non-negative integer solutions of such a system are additively generated by a finite set of solutions.

Lemma 3 Let $\mathfrak{A}=\left(a_{i j}\right)$ be an integer $(n \times m)$-matrix. Set

$$
K=\left(\max _{i=1, \ldots, n} \sum_{j=1}^{m} a_{i j}^{2}\right)^{1 / 2}
$$

There is a set $\left\{\mathfrak{x}_{1}, \ldots \mathfrak{x}_{p}\right\}$ of non-negative integer vectors such that $\mathfrak{A} \cdot \mathfrak{x}_{i}=0$ for any $i=1, \ldots, p$, the components of $\mathfrak{x}_{i}$ are bounded from above by $m K^{m}$, and any non-negative integer solution $\mathfrak{x}$ of $\mathfrak{A} \cdot \mathfrak{x}=0$ can be written as a sum $\mathfrak{x}=\sum k_{i} \mathfrak{x}_{i}$ with non-negative integers $k_{1}, \ldots, k_{p}$.

The set $\left\{\mathfrak{x}_{1}, \ldots \mathfrak{x}_{p}\right\}$ is called Hilbert base for $\mathfrak{A}$, if $p$ is minimal.

As in the previous section, if $F \subset N$ is a $2-$ normal surface and $\mathfrak{x}_{N}(F)$ is a sum of two non-negative integer solutions of $\mathfrak{A} \cdot \mathfrak{x}=0$ then there are 2 -normal surfaces $F^{\prime}, F^{\prime \prime} \subset N$ with $F=F^{\prime}+F^{\prime \prime}$. Thus the surfaces $F_{1}, \ldots, F_{q} \subset N$ that correspond to Hilbert base vectors satisfying the compatibility condition additively generate the set of all 2 -normal surfaces in $N$. 
It remains to bound $\left\|F_{i}\right\|$, for $i=1, \ldots, q$. Since $F_{i}$ is $2-$ normal and any edge of $\mathcal{T}$ is of degree $\geq 3$, we have $\left\|F_{i}\right\| \leq \frac{8}{3} \#\left(F_{i} \backslash \mathcal{T}^{2}\right)$. By the elimination process in the proof of Lemma 2, any component of $\overline{\mathfrak{x}}_{N}\left(F_{i}\right)$ that corresponds to a parallelity region of $N$ is a sum of at most four components of $\mathfrak{x}_{N}\left(F_{i}\right)$. By the bound for the components of $\mathfrak{x}_{N}\left(F_{i}\right)$ in Lemma 3 (with $m=m(N)$ and $\left.K^{2}=8\right)$ and our bounds for $m(N)$ and $\bar{m}(N)$, we obtain

$$
\begin{aligned}
\left\|F_{i}\right\| & \leq \frac{8}{3} \cdot(m(N)+4 \bar{m}(N)) \cdot\left(m(N) \cdot 2^{\frac{3}{2} m(N)}\right) \\
& \leq \frac{8}{3} \cdot\left(10 t(\mathcal{T})+\frac{2}{3}\|\partial N\| t(\mathcal{T})\right) \cdot 10 t(\mathcal{T}) \cdot 2^{15 t(\mathcal{T})} \\
& <(300+20\|\partial N\|) \cdot t(\mathcal{T})^{2} \cdot 2^{15 t(\mathcal{T})} .
\end{aligned}
$$

Using $t(\mathcal{T}) \geq 5$ and $\|\partial N\|>0$, we obtain $\left\|F_{i}\right\|<\|\partial N\| \cdot 2^{18 t(\mathcal{T})}$.

\section{Maximal systems of 1-normal spheres}

Let $\mathcal{T}$ be a triangulation of a closed orientable 3-manifold $M$. By Proposition 2, there is a system $\Sigma \subset M$ of $\leq 10 t(\mathcal{T})$ pairwise disjoint 1-normal spheres, such that any 1 -normal sphere in $M \backslash \Sigma$ is isotopic $\bmod \mathcal{T}^{2}$ to a component of $\Sigma$. We call such a system maximal. It is not obvious how to construct $\Sigma$, in particular how to estimate $\|\Sigma\|$ in terms of $t(\mathcal{T})$. This section is devoted to a solution of this problem.

Construction 1 Set $\Sigma_{1}=\partial U\left(\mathcal{T}^{0}\right)$ and $N_{1}=M \backslash U\left(\mathcal{T}^{0}\right)$. Let $i \geq 1$. If there is a 1-normal fundamental projective plane $P_{i} \subset N_{i}$ then set $\Sigma_{i+1}=\Sigma_{i} \cup 2 P_{i}$ and $N_{i+1}=N_{i} \backslash U\left(P_{i}\right)$. Otherwise, if there is a 1-normal fundamental sphere $S_{i} \subset N_{i}$ that is not isotopic $\bmod \mathcal{T}^{2}$ to a component of $\Sigma_{i}$, then set $\Sigma_{i+1}=$ $\Sigma_{i} \cup S_{i}$ and $N_{i+1}=N_{i} \backslash U\left(S_{i}\right)$. Otherwise, set $\Sigma=\Sigma_{i}$.

Since $M$ is orientable, a projective plane $P_{i}$ is one-sided and $2 P_{i}$ is a sphere. By Proposition 2 and since embedded spheres are two-sided in $M$, the iteration stops for some $i<10 t(\mathcal{T})$.

Lemma $4\|\Sigma\|<2^{185 t(\mathcal{T})^{2}}$.

Proof In Construction 1, we have

$$
\begin{aligned}
\left\|\Sigma_{i+1}\right\| & <\left\|\Sigma_{i}\right\|+2\left\|\Sigma_{i}\right\| \cdot 2^{18 t(\mathcal{T})} \\
& <\left\|\Sigma_{i}\right\| \cdot 2^{18 t(\mathcal{T})+2}
\end{aligned}
$$

Geometry \& Topology, Volume 5 (2001) 
by Theorem 3 . The iteration stops after $<10 t(\mathcal{T})$ steps, thus

$$
\|\Sigma\|<\left\|\Sigma_{1}\right\| \cdot 2^{180 t(\mathcal{T})^{2}+20 t(\mathcal{T})} \leq\left\|\Sigma_{1}\right\| \cdot 2^{184 t(\mathcal{T})^{2}},
$$

using $t(\mathcal{T}) \geq 5$. Since $\left\|\partial U\left(\mathcal{T}^{0}\right)\right\|$ equals twice the number of edges of $\mathcal{T}$, we have $\left\|\Sigma_{1}\right\| \leq 4 t(\mathcal{T})$, and the lemma follows.

Lemma $5 \quad \Sigma$ is maximal.

Proof It is to show that any 1 -normal sphere $S \subset M \backslash U(\Sigma)$ is isotopic mod $\mathcal{T}^{2}$ to a component of $\Sigma$. Let $N$ be the component of $M \backslash U(\Sigma)$ that contains $S$. If $N$ contains a 1-normal fundamental projective plane $P$, then $N=U(P)$ by Construction 1. Thus $S=2 P=\partial N$, which is isotopic $\bmod \mathcal{T}^{2}$ to a component of $\Sigma$. Hence we can assume that $N$ does not contain a 1 -normal fundamental projective plane.

We express $S$ as a sum $\sum_{i=1}^{q} k_{i} F_{i}$ of fundamental surfaces in $N$. Since $\chi(S)=2$ and the Euler characteristic is additive, one of the fundamental surfaces in the sum, say, $F_{1}$ with $k_{1}>0$, has positive Euler characteristic. It is not a projective plane by the preceding paragraph, thus it is a sphere. By construction of $\Sigma$, the sphere $F_{1}$ is isotopic mod $\mathcal{T}^{2}$ to a component of $\Sigma$, thus it is parallel to a component of $\partial N$. Hence $F_{1}$ is disjoint to any 1 -normal surface in $N$, up to isotopy $\bmod \mathcal{T}^{2}$. Thus $S$ is the disjoint union of $k_{1} F_{1}$ and $\sum_{i=2}^{q} k_{i} F_{i}$. Since $S$ is connected, it follows $S=F_{1}$. Thus $S$ is isotopic $\bmod \mathcal{T}^{2}$ to a component of $\Sigma$.

We will extend $\Sigma$ to a system $\tilde{\Sigma}$ of $2-$ normal spheres. To define $\tilde{\Sigma}$, we need a lemma about 2 -normal spheres in the complement of $\Sigma$.

Lemma 6 Let $N$ be a component of $M \backslash U(\Sigma)$. Assume that there is a 2 -normal sphere in $N$ with exactly one octagon. Then there is a 2-normal fundamental sphere $F \subset N$ with exactly one octagon and $\|F\|<2^{189 t(\mathcal{T})^{2}}$.

Proof Let $S \subset N$ be a $2-$ normal sphere with exactly one octagon. If $N$ contains a 1-normal fundamental projective plane $P$, then $N=U(P)$ by Construction 1 , and any pre-normal surface in $N$ is a multiple of $P$, i.e., is $1-$ normal. Thus since $S \subset N$ is not 1 -normal, there is no 1 -normal fundamental projective plane in $N$.

We write $S$ as a sum of $2-$ normal fundamental surfaces in $N$. Since $S$ has exactly one octagon, exactly one summand is not $1-$ normal. Since any projective plane in the sum is not 1 -normal by the preceding paragraph, at most one 
summand is a projective plane. Since $\chi(S)=2$ and the Euler characteristic is additive, it follows that one of the fundamental surfaces in the sum is a sphere $F$.

Assume that $F$ is 1 -normal, i.e., $S \neq F$. The construction of $\Sigma$ implies that $F$ is isotopic $\bmod \mathcal{T}^{2}$ to a component of $\partial N$. Thus it is disjoint to any $2-$ normal surface in $N$. Therefore $S$ is a disjoint union of a multiple of $F$ and of a $2-$ normal surface with exactly one octagon, which is a contradiction since $S$ is connected. Hence $F$ contains the octagon of $S$. We have $\|F\|<\|\Sigma\| \cdot 2^{18 t(\mathcal{T})}$ by Theorem 3 . The claim follows with Lemma 4 and $t(\mathcal{T}) \geq 5$.

The preceding lemma assures that the following construction works.

Construction 2 For any connected component $N$ of $M \backslash U(\Sigma)$ that contains a 2-normal sphere with exactly one octagon, choose a 2-normal sphere $F_{N} \subset N$ with exactly one octagon and $\|F\|<2^{189 t(\mathcal{T})^{2}}$. Set

$$
\tilde{\Sigma}=\Sigma \cup \bigcup_{N} F_{N}
$$

Since $\#(\tilde{\Sigma}) \leq 10 t(\mathcal{T})$ by Proposition 2 , it follows $\|\tilde{\Sigma}\|<10 t(\mathcal{T}) \cdot 2^{189 t(\mathcal{T})^{2}}<$ $2^{190 t(\mathcal{T})^{2}}$.

\section{Almost $k$-normal surfaces and split equivalence}

We shall need a generalization of the notion of $k$-normal surfaces. Let $M$ be a closed connected orientable 3 -manifold with a triangulation $\mathcal{T}$.

Definition 6 A closed embedded surface $S \subset M$ transversal to $\mathcal{T}^{2}$ is almost $k$-normal, if

(1) $S \cap \mathcal{T}^{2}$ is a union of normal arcs and of circles in $\mathcal{T}^{2} \backslash \mathcal{T}^{1}$, and

(2) for any tetrahedron $t$ of $\mathcal{T}$, any edge $e$ of $t$ and any component $\zeta$ of $S \cap \partial t$ holds $\#(\zeta \cap e) \leq k$.

Our definition is similar to Matveev's one in [16]. Note that there is a related but different definition of "almost normal" surfaces due to Rubinstein [19]. Any surface in $M$ disjoint to $\mathcal{T}^{1}$ is almost 1-normal. Any almost $k$-normal surface that meets $\mathcal{T}^{1}$ can be seen as a $k$-normal surface with several disjoint small tubes attached in $M \backslash \mathcal{T}^{1}$, see [16]. The tubes can be nested. Of course there 
are many ways to add tubes to a $k$-normal surface. We shall develop tools to deal with this ambiguity.

Let $S \subset M$ be an almost $k$-normal surface. By definition, the connected components of $S \cap \mathcal{T}^{2}$ that meet $\mathcal{T}^{1}$ are formed by normal arcs. Thus these components define a pre-normal surface $S^{\times}$, that is obviously $k$-normal. It is determined by $S \cap \mathcal{T}^{1}$, according to Lemma 1. A disc $D \subset M \backslash \mathcal{T}^{1}$ with $\partial D \subset S$ is called a splitting disc for $S$. One obtains $S^{\times}$by splitting $S$ along splitting discs for $S$ that are disjoint to $\mathcal{T}^{2}$, and isotopy $\bmod \mathcal{T}^{1}$.

If two almost $k$-normal surfaces $S_{1}, S_{2}$ satisfy $S_{1}^{\times}=S_{2}^{\times}$, then $S_{1}$ and $S_{2}$ differ only by the choice of tubes. This gives rise to the following equivalence relation.

Definition 7 Two embedded surfaces $S_{1}, S_{2} \subset M$ transversal to $\mathcal{T}^{2}$ are split equivalent if $S_{1} \cap \mathcal{T}^{1}=S_{2} \cap \mathcal{T}^{1}$ (up to isotopy $\bmod \mathcal{T}^{2}$ ).

If two almost $k$-normal surfaces $S_{1}, S_{2} \subset M$ are split equivalent, then $S_{1}^{\times}=$ $S_{2}^{\times}$, up to isotopy mod $\mathcal{T}^{2}$. In particular, two $k$-normal surfaces are split equivalent if and only if they are isotopic $\bmod \mathcal{T}^{2}$.

Definition 8 If $S \subset M$ is an almost $k$-normal surface and $S^{\times}$is the disjoint union of $k$-normal surfaces $S_{1}, \ldots, S_{n}$, then we call $S$ a tube sum of $S_{1}, \ldots, S_{n}$. We denote the set of all tube sums of $S_{1}, \ldots, S_{n}$ by $S_{1} \circ \ldots \circ S_{n}$.

Definition 9 Let $S=S_{1} \cup \cdots \cup S_{n} \subset M$ be a surface transversal to $\mathcal{T}^{2}$ with $n$ connected components, and let $\Gamma \subset M \backslash \mathcal{T}^{1}$ be a system of disjoint simple arcs with $\Gamma \cap S=\partial \Gamma$. For any $\operatorname{arc} \gamma$ in $\Gamma$, one component of $\partial U(\gamma) \backslash S$ is an annulus $A_{\gamma}$. The surface

$$
S^{\Gamma}=(S \backslash U(\Gamma)) \cup \bigcup_{\gamma \subset \Gamma} A_{\gamma}
$$

is called the tube sum of $S_{1}, \ldots, S_{n}$ along $\Gamma$.

If $S_{1}, \ldots, S_{n}$ are $k$-normal, then $S^{\Gamma} \in S_{1} \circ \cdots \circ S_{n}$.

We recall the concept of impermeable surfaces, that is central in the study of almost 2-normal surfaces (see [22],[16]). Fix a vertex $x_{0} \in \mathcal{T}^{0}$. Let $S \subset M$ be a connected embedded surface transversal to $\mathcal{T}$. If $S$ splits $M$ into two pieces, then let $B^{+}(S)$ denote the closure of the component of $M \backslash S$ that contains $x_{0}$, and let $B^{-}(S)$ denote the closure of the other component. We do not include $x_{0}$ in the notation " $B^{+}(S)$ ", since in our applications the choice of $x_{0}$ plays no essential role. 
Definition 10 Let $S \subset M$ be a connected embedded surface transversal to $\mathcal{T}^{2}$. Let $\alpha \subset \mathcal{T}^{1} \backslash \mathcal{T}^{0}$ and $\beta \subset S$ be embedded arcs with $\partial \alpha=\partial \beta$. A closed embedded disc $D \subset M$ is a compressing disc for $S$ with string $\alpha$ and base $\beta$, if $\partial D=\alpha \cup \beta$ and $D \cap \mathcal{T}^{1}=\alpha$. If, moreover, $D \cap S=\beta$, then we call $D$ a bond of $S$.

Let $S \subset M$ be a connected embedded surface that splits $M$ and let $D$ be a compressing disc for $S$ with string $\alpha$. If the germ of $\alpha$ in $\partial \alpha$ is contained in $B^{+}(S)$ (resp. $B^{-}(S)$ ), then $D$ is upper (resp. lower). Let $D_{1}, D_{2}$ be upper and lower compressing discs for $S$ with strings $\alpha_{1}, \alpha_{2}$. If $D_{1} \subset D_{2}$ or $D_{2} \subset D_{1}$, then $D_{1}$ and $D_{2}$ are nested. If $D_{1} \cap D_{2} \subset \partial \alpha_{1} \cap \partial \alpha_{2}$, then $D_{1}$ and $D_{2}$ are independent from each other.

Upper and lower compressing discs that are independent from each other meet in at most one point.

Definition 11 Let $S \subset M$ be a connected embedded surface that is transversal to $\mathcal{T}^{2}$ and splits $M$. If $S$ has both upper and lower bonds, but no pair of nested or independent upper and lower compressing discs, then $S$ is impermeable.

Note that the impermeability of $S$ does not change under an isotopy of $S$ $\bmod \mathcal{T}^{1}$. The next two claims state a close relationship between impermeable surfaces and (almost) 2-normal surfaces. By an octagon of an almost 2-normal surface $S \subset M$ in a tetrahedron $t$, we mean a circle in $S \cap \partial t$ formed by eight normal arcs. This corresponds to an octagon of $S^{\times}$in the sense of Figure 2.

Proposition 3 Any impermeable surface in $M$ is isotopic mod $\mathcal{T}^{1}$ to an almost 2-normal surface with exactly one octagon.

Proposition 4 A connected 2-normal surface that splits $M$ and contains exactly one octagon is impermeable.

We shall need these statements later. As the author found only parts of the proofs in the literature (see [22],[16]), he includes proofs in Section 9.

We end this section with the definition of $\mathcal{T}^{1}$-Morse embeddings and with the notion of thin position. Let $S$ be a closed 2-manifold and let $H: S \times I \rightarrow M$ be a tame embedding. For $\xi \in I$, set $H_{\xi}=H(S \times \xi)$.

Definition 12 An element $\xi \in I$ is a critical parameter of $H$ and a point $x \in H_{\xi}$ is a critical point of $H$ with respect to $\mathcal{T}^{1}$, if $x$ is a vertex of $\mathcal{T}$ or $x$ is a point of tangency of $H_{\xi}$ to $\mathcal{T}^{1}$. 
Definition 13 We call $H$ a $\mathcal{T}^{1}$-Morse embedding, if it has finitely many critical parameters, to any critical parameter belongs exactly one critical point, and for any critical point $x \in \mathcal{T}^{1} \backslash \mathcal{T}^{0}$ corresponding to a critical parameter $\xi$, one component of $U(x) \backslash H_{\xi}$ is disjoint to $\mathcal{T}^{1}$. The number of critical points with respect to $\mathcal{T}^{1}$ of a $\mathcal{T}^{1}$-Morse embedding $H$ is denoted by $c\left(H, \mathcal{T}^{1}\right)$.

The last condition in the definition of $\mathcal{T}^{1}$-Morse embeddings means that any critical point of $H$ is a vertex of $\mathcal{T}$ or a local maximum resp. minimum of an edge of $\mathcal{T}$.

Definition 14 Let $F$ be a closed surface, let $J: F \times I \rightarrow M$ be a $\mathcal{T}^{1}$-Morse embedding, and let $\xi_{1}, \ldots, \xi_{r} \in I$ be the critical parameters of $J$ with respect to $\mathcal{T}^{1}$. The complexity $\kappa(J)$ of $J$ is defined as

$$
\kappa(J)=\#\left(\mathcal{T}^{1} \backslash\left(\bigcup_{i=1}^{r} J_{\xi_{i}}\right)\right) .
$$

If $\kappa(J)$ is minimal among all $\mathcal{T}^{1}$-Morse embeddings with the property $\mathcal{T}^{1} \subset$ $J(F \times I)$, then $J$ is said to be in thin position with respect to $\mathcal{T}^{1}$. This notion was introduced for foliations of 3-manifolds by Gabai [5], was applied by Thompson [22] for her recognition algorithm of $S^{3}$, and was also used in the study of Heegaard surfaces by Scharlemann and Thompson [20].

If $J(F \times \xi)$ splits $M$ and has a pair of nested or independent upper and lower compressing discs $D_{1}, D_{2}$, then an isotopy of $J$ along $D_{1} \cup D_{2}$ decreases $\kappa(J)$, see [16], [22]. We obtain the following claim.

Lemma 7 Let $J: F \times I \rightarrow M$ be a $\mathcal{T}^{1}$-Morse embedding in thin position and let $\xi \in I$ be a non-critical parameter of $J$. If $J(F \times \xi)$ has both upper and lower bonds, then $J(F \times \xi)$ is impermeable.

\section{Compressing and splitting discs}

Let $M$ be a closed connected 3-manifold with a triangulation $\mathcal{T}$. In the lemmas that we prove in this section, we state technical conditions for the existence of compressing and splitting discs for a surface.

Lemma 8 Let $S_{1}, \ldots, S_{n} \subset M$ be embedded surfaces transversal to $\mathcal{T}^{2}$ and let $S$ be the tube sum of $S_{1}, \ldots, S_{n}$ along a system $\Gamma \subset M \backslash \mathcal{T}^{1}$ of arcs. Assume that $S$ splits $M$, and $\Gamma \subset B^{-}(S)$. If none of $S_{1}, \ldots, S_{n}$ has a lower compressing disc, then $S$ has no lower compressing disc. 
Proof Set $\Sigma=S_{1} \cup \cdots \cup S_{n}$. Let $D \subset M$ be a lower compressing disc for $S$. One can assume that a collar of $\partial D \cap S$ in $D$ is contained in $B^{-}(S)$. Then, since by hypothesis $U(\Gamma) \cap \Sigma \subset B^{-}(S)$, any point in $\partial D \cap U(\Gamma) \cap \Sigma$ is endpoint of an arc in $D \cap \Sigma$. Therefore there is a sub-disc $D^{\prime} \subset D$, bounded by parts of $\partial D$ and of $\operatorname{arcs}$ in $D \cap \Sigma$, that is a lower compressing disc for one of $S_{1}, \ldots, S_{n}$.

Lemma 9 Let $S \subset M$ be a surface transversal to $\mathcal{T}^{2}$ with upper and lower compressing discs $D_{1}, D_{2}$ such that $\partial\left(D_{1} \cap D_{2}\right) \subset \partial D_{2} \cap S$. Assume either that $\left(\partial D_{1}\right) \cap D_{2} \subset \mathcal{T}^{1}$ or that there is a splitting disc $D_{m}$ for $S$ such that $D_{1} \cap D_{m}=\partial D_{1} \cap \partial D_{m}=\{x\}$ is a single point and $D_{2} \cap D_{m}=\emptyset$. Then $S$ has a pair of independent or nested upper and lower compressing discs.

Proof If $D_{1} \cap D_{2} \cap \mathcal{T}^{1}$ comprises more than a single point then the string of $D_{2}$ is contained in the string of $D_{1}$. Thus $D_{1} \cap S$ contains an arc different from the base of $D_{1}$, bounding in $D_{1}$ a lower compressing disc, that forms with $D_{1}$ a pair of nested upper and lower compressing discs for $S$.

Assume that a component $\gamma$ of $D_{1} \cap D_{2}$ is a circle. Then there are discs $D_{1}^{\prime} \subset D_{1}$ and $D_{2}^{\prime} \subset D_{2}$ with $\partial D_{1}^{\prime}=\partial D_{2}^{\prime}=\gamma$. Since $\partial\left(D_{1} \cap D_{2}\right) \subset \partial D_{2}$, $D_{2}^{\prime}$ does not contain arcs of $D_{1} \cap D_{2}$. Thus if we choose $\gamma$ innermost in $D_{2}$, then $D_{1} \cap D_{2}^{\prime}=\gamma$. By cut-and-paste of $D_{1}$ along $D_{2}^{\prime}$, one reduces the number of circle components in $D_{1} \cap D_{2}$. Therefore we assume by now that $D_{1} \cap D_{2}$ consists of isolated points in $\partial D_{1} \cap \partial D_{2}$ and of arcs that do not meet $\partial D_{1}$.

Assume that there is a point $y \in\left(\partial D_{1} \cap \partial D_{2}\right) \backslash \mathcal{T}^{1}$. Then there is an arc $\gamma \subset \partial D_{1}$ with $\partial \gamma=\{x, y\}$. Without assumption, let $\gamma \cap D_{2}=\{y\}$. Let $A$ be the closure of the component of $U(\gamma) \backslash\left(D_{1} \cup D_{2} \cup D_{m}\right)$ whose boundary contains arcs in both $D_{2}$ and $D_{m}$. Define $D_{2}^{*}=\left(\left(D_{2} \cup D_{m}\right) \backslash U(\gamma)\right) \cup A$, that is to say, $D_{2}^{*}$ is the connected sum of $D_{2}$ and $D_{m}$ along $\gamma$. By construction, $\left(D_{1} \cap D_{2}^{*}\right) \backslash \partial D_{1}=\left(D_{1} \cap D_{2}\right) \backslash \partial D_{1}$, and $\#\left(D_{1} \cap D_{2}^{*}\right)<\#\left(D_{1} \cap D_{2}\right)$. In that way, we remove all points of intersection of $\left(\partial D_{1} \cap D_{2}\right) \backslash \mathcal{T}^{1}$. Thus by now we can assume that $D_{1} \cap D_{2}$ consists of arcs in $D_{1}$ that do not meet $\partial D_{1}$, and possibly of a single point in $\mathcal{T}^{1}$.

Let $\gamma \subset D_{1} \cap D_{2}$ be an outermost arc in $D_{2}$, that is to say, $\gamma \cup \partial D_{2}$ bounds a $\operatorname{disc} D^{\prime} \subset D_{2} \backslash \mathcal{T}^{1}$ with $D_{1} \cap D^{\prime}=\gamma$. We move $D_{1}$ away from $D^{\prime}$ by an isotopy $\bmod \mathcal{T}^{1}$ and obtain a compressing disc $D_{1}^{*}$ for $S$ with $D_{1}^{*} \cap D_{2}=\left(D_{1} \cap D_{2}\right) \backslash \gamma$. In that way, we remove all arcs of $D_{1} \cap D_{2}$ and finally get a pair of independent upper and lower compressing discs for $S$.

Lemma 10 Let $S \subset M$ be an almost 1-normal surface. If $S$ has a compressing disc, then $S$ is isotopic $\bmod \mathcal{T}^{1}$ to an almost 1-normal surface with 
a compressing disc contained in a single tetrahedron. In particular, $S$ is not $1-$ normal.

Proof Let $D$ be a compressing disc for $S$. Choose $S$ and $D$ up to isotopy of $S \cup D \bmod \mathcal{T}^{1}$ so that $S$ is almost 1 -normal and $\#\left(D \cap \mathcal{T}^{2}\right)$ is minimal. Choose an innermost component $\gamma \subset\left(D \cap \mathcal{T}^{2}\right)$, which is possible as $D \cap \mathcal{T}^{2} \neq \emptyset$. There is a closed tetrahedron $t$ of $\mathcal{T}$ and a component $C$ of $D \cap t$ that is a disc, such that $\gamma=C \cap \partial t$. Let $\sigma$ be the closed 2-simplex of $\mathcal{T}$ that contains $\gamma$. We obtain three cases.

(1) Let $\gamma$ be a circle, thus $\partial C=\gamma$. Then there is a disc $D^{\prime} \subset \sigma$ with $\partial D^{\prime}=\gamma$ and a ball $B \subset t$ with $\partial B=C \cup D^{\prime}$. By an isotopy $\bmod \mathcal{T}^{1}$ with support in $U(B)$, we move $S \cup D$ away from $B$, obtaining a surface $S^{*}$ with a compressing disc $D^{*}$. If $S^{*}$ is almost 1 -normal, then we obtain a contradiction to our choice as $\#\left(D^{*} \cap \mathcal{T}^{2}\right)<\#\left(D \cap \mathcal{T}^{2}\right)$.

(2) Let $\gamma$ be an arc with endpoints in a single component $c$ of $S \cap \sigma$. Since $S$ has no returns, $\gamma$ is not the string of $D$. We apply to $S \cup D$ an isotopy $\bmod \mathcal{T}^{1}$ with support in $U(C)$ that moves $C$ into $U(C) \backslash t$, and obtain a surface $S^{*}$ with a compressing disc $D^{*}$. If $S^{*}$ is almost 1 -normal, then we obtain a contradiction to our choice as $\#\left(D^{*} \cap \mathcal{T}^{2}\right)<\#\left(D \cap \mathcal{T}^{2}\right)$.

(3) Let $\gamma$ be an arc with endpoints in two different components $c_{1}, c_{2}$ of $S \cap \sigma$. If both $c_{1}$ and $c_{2}$ are normal arcs, then set $C^{\prime}=C, c_{1}^{\prime}=c_{1}$ and $c_{2}^{\prime}=c_{2}$. If, say, $c_{1}$ is a circle, then we move $S \cup D$ away from $C$ by an isotopy $\bmod \mathcal{T}^{1}$ with support in $U(C)$. If the resulting surface $S^{*}$ is still almost 1 -normal, then we obtain a contradiction to the choice of $D$.

In either case, $S^{*}$ is not almost 1-normal, i.e., the isotopy introduces a return. Therefore there is a component of $C \backslash S$ with closure $C^{\prime}$ such that $\partial C^{\prime} \cap S$ connects two normal arcs $c_{1}^{\prime}, c_{2}^{\prime}$ of $S \cap \sigma$.

Let $\gamma^{\prime}=C^{\prime} \cap \sigma$. Up to isotopy of $C^{\prime} \bmod \mathcal{T}^{2}$ that is fixed on $\partial C^{\prime} \cap S$, we assume that $\gamma^{\prime} \cap\left(c_{1}^{\prime} \cup c_{2}^{\prime}\right) \subset \partial \gamma^{\prime}$. There is an arc $\alpha$ contained in an edge of $\sigma$ with $\partial \alpha \subset c_{1}^{\prime} \cup c_{2}^{\prime}$. For $i \in\{1,2\}$, there is an arc $\beta_{i} \subset c_{i}^{\prime}$ that connects $\alpha \cap c_{i}^{\prime}$ with $\gamma^{\prime} \cap c_{i}^{\prime}$. The circle $\alpha \cup \beta_{1} \cup \beta_{2} \cup \gamma^{\prime}$ bounds a closed disc $D^{\prime} \subset \sigma$. Eventually $D^{\prime} \cup C^{\prime}$ is a compressing disc for $S$ contained in a single tetrahedron.

Lemma 11 Let $S \subset M$ be a 1-normal surface and let $D$ be a splitting disc for $S$. Then, $(D, \partial D)$ is isotopic in $\left(M \backslash \mathcal{T}^{1}, S \backslash \mathcal{T}^{1}\right)$ to a disc embedded in $S$.

Proof We choose $D$ up to isotopy of $(D, \partial D)$ in $\left(M \backslash \mathcal{T}^{1}, S \backslash \mathcal{T}^{1}\right)$ so that $\left(\#\left((\partial D) \cap \mathcal{T}^{2}\right), \#\left(D \cap \mathcal{T}^{2}\right)\right)$ is minimal in lexicographic order. Assume that 
$\partial D \cap \mathcal{T}^{2} \neq \emptyset$. Then, there is a tetrahedron $t$, a 2 -simplex $\sigma \subset \partial t$, a component $K$ of $S \cap t$, and a component $\gamma$ of $\partial D \cap K$ with $\partial \gamma \subset \sigma$. Since $S$ is 1-normal, the closure $D^{\prime}$ of one component of $K \backslash \gamma$ is a disc with $\partial D^{\prime} \subset \gamma \cup \sigma$. By choosing $\gamma$ innermost in $D$, we can assume that $D^{\prime} \cap \partial D=\gamma$. An isotopy of $(D, \partial D)$ in $\left(M \backslash \mathcal{T}^{1}, S \backslash \mathcal{T}^{1}\right)$ with support in $U\left(D^{\prime}\right)$, moving $\partial D$ away from $D^{\prime}$, reduces $\#\left(\partial D \cap \mathcal{T}^{2}\right)$, in contradiction to our choice. Thus $\partial D \cap \mathcal{T}^{2}=\emptyset$.

Now, assume that $D \cap \mathcal{T}^{2} \neq \emptyset$. Then, there is a tetrahedron $t$, a 2-simplex $\sigma \subset \partial t$, and a disc component $C$ of $D \cap t$, such that $C \cap \sigma=\partial C$ is a single circle. There is a ball $B \subset t$ bounded by $C$ and a disc in $\sigma$. An isotopy of $D$ with support in $U(B)$, moving $C$ away from $t$, reduces $\#\left(D \cap \mathcal{T}^{2}\right)$, in contradiction to our choice. Thus $D$ is contained in a single tetrahedron $t$. Since $S$ is 1-normal, $\partial D$ bounds a disc $D^{\prime}$ in $S \cap t$. An isotopy with support in $t$ that is constant on $\partial D$ moves $D$ to $D^{\prime}$, which yields the lemma.

Corollary 1 Let $S_{0} \subset M$ be a 1-normal sphere that splits $M$, and let $S \subset$ $B^{-}\left(S_{0}\right)$ be an almost 1-normal sphere disjoint to $S_{0}$ that is split equivalent to $S_{0}$. Then there is a $\mathcal{T}^{1}$-Morse embedding $J: S^{2} \times I \rightarrow M$ with $J\left(S^{2} \times I\right)=$ $B^{+}(S) \cap B^{-}\left(S_{0}\right)$ and $c\left(J, \mathcal{T}^{1}\right)=0$.

Proof Let $X$ be a graph isomorphic to $S_{0} \cap \mathcal{T}^{2}$. Since $S^{\times}$is a copy of $S_{0}$, there is an embedding $\varphi: X \times I \rightarrow B^{+}(S) \cap B^{-}\left(S_{0}\right)$ with $\varphi\left(X^{0} \times I\right)=\varphi(X \times I) \cap \mathcal{T}^{1}$, $\varphi(X \times 0)=S_{0} \cap \mathcal{T}^{2}=S_{0} \cap \varphi(X \times I)$, and $\varphi(X \times 1)$ is the union of the normal $\operatorname{arcs}$ in $S$.

Let $\gamma \subset S \cap \varphi(X \times I)$ be a circle that does not meet $\mathcal{T}^{1}$. Then, $\gamma$ bounds a disc $D \subset \varphi(X \times I) \backslash \mathcal{T}^{1}$. The two components of $S \backslash \gamma$ are discs. One of them is disjoint to $\mathcal{T}^{1}$, since otherwise the disc $D$ would give rise to a splitting disc for $S^{\times}=S_{0}$ that is not isotopic mod $\mathcal{T}^{1}$ to a sub-disc of $S_{0}$, in contradiction to the preceding lemma. Thus by cut-and-paste along sub-discs of $S \backslash \mathcal{T}^{1}$, we can assume that additionally $S \cap \varphi(X \times I)=\varphi(X \times 1)$.

Let $\gamma \subset X$ be a circle so that $\varphi(\gamma \times 0)$ is contained in the boundary of a tetrahedron of $\mathcal{T}$. Since $S_{0}$ is 1 -normal, $\varphi(\gamma \times 0)$ bounds an open disc in $S_{0} \backslash \mathcal{T}^{2}$. By the same argument as in the preceding paragraph, $\varphi(\gamma \times 1)$ bounds an open disc in $S \backslash \mathcal{T}^{1}$. One easily verifies that these two discs together with $\varphi(\gamma \times I)$ bound a ball in $B^{+}(S) \cap B^{-}\left(S_{0}\right)$ disjoint to $\mathcal{T}^{1}$. Hence $\left(B^{+}(S) \cap\right.$ $\left.B^{-}\left(S_{0}\right)\right) \backslash U(\varphi(X \times I))$ is a disjoint union of balls in $M \backslash \mathcal{T}^{1}$, and this implies the existence of $J$. 


\section{$7 \quad$ Reduction of surfaces}

Let $M$ be a closed connected orientable 3-manifold with a triangulation $\mathcal{T}$. In this section, we show how to get isotopies of embedded surfaces under which the number of intersections with $\mathcal{T}^{1}$ is monotonely non-increasing.

Definition 15 Let $S \subset M$ be a connected embedded surface that is transversal to $\mathcal{T}^{2}$ and splits $M$. Let $D$ be an upper (resp. lower) bond of $S$, set $D_{1}=U(D) \cap S$, and set $D_{2}=B^{+}(S) \cap \partial U(D)\left(\right.$ resp. $\left.D_{2}=B^{-}(S) \cap \partial U(D)\right)$. An elementary reduction along $D$ transforms $S$ to the surface $\left(S \backslash D_{1}\right) \cup D_{2}$. Upper (resp. lower) reductions of $S$ are the surfaces that are obtained from $S$ by a sequence of elementary reductions along upper (resp. lower) bonds.

If $S^{\prime}$ is an upper or lower reduction of $S$, then $\left\|S^{\prime}\right\| \leq\|S\|$ with equality if and only if $S=S^{\prime}$. Obviously $S$ is isotopic to $S^{\prime}$, such that $\|\cdot\|$ is monotonely non-increasing under the isotopy. If $\alpha \subset \mathcal{T}^{1} \backslash \mathcal{T}^{0}$ is an arc with $\partial \alpha \subset S^{\prime}$, then also $\partial \alpha \subset S$. It is easy to see that if $S^{\prime}$ has a lower compressing disc and is an upper reduction of $S$, then also $S$ has a lower compressing disc.

We will construct surfaces with almost 1-normal upper or lower reductions. Let $N \subset M$ be a 3-dimensional sub-manifold, such that $\partial N$ is pre-normal. Let $S \subset N$ be an embedded surface transversal to $\mathcal{T}^{2}$ that splits $M$ and has no lower compressing disc.

Lemma 12 Suppose that there is a system $\Gamma \subset N \backslash \mathcal{T}^{1}$ of arcs such that $S^{\Gamma} \subset N$ is connected, $\Gamma \subset B^{-}\left(S^{\Gamma}\right)$, and $\partial N \cap B^{+}\left(S^{\Gamma}\right)$ is 1-normal.

If, moreover, $\Gamma$ and an upper reduction $S^{\prime} \subset N$ of $S^{\Gamma}$ are chosen so that $\left\|S^{\prime}\right\|$ is minimal, then $S^{\prime}$ is almost 1-normal.

Proof By hypothesis, $\Gamma \subset B^{-}\left(S^{\Gamma}\right)$, and $S$ has no lower compressing discs. Thus by Lemma $8, S^{\Gamma}$ has no lower compressing discs. Therefore its upper reduction $S^{\prime}$ has no lower compressing discs.

Assume that $S^{\prime}$ is not almost $1-$ normal. Then $S^{\prime}$ has a compressing disc $D^{\prime}$ that is contained in a single tetrahedron $t$ (see [16]), with string $\alpha^{\prime}$ and base $\beta^{\prime}$. Since $S^{\prime}$ has no lower compressing discs, $D^{\prime}$ is upper and does not contain proper compressing sub-discs. Thus $\alpha^{\prime} \cap S^{\prime}=\partial \alpha^{\prime}$, i.e., all components of $\left(D^{\prime} \cap S^{\prime}\right) \backslash \beta^{\prime}$ are circles. Since $\partial N$ is pre-normal, $\partial N \backslash \mathcal{T}^{2}$ is a disjoint union of discs. Therefore, since $D^{\prime}$ is contained in a single tetrahedron, we can assume by isotopy of $D^{\prime} \bmod \mathcal{T}^{2}$ that $D^{\prime} \cap \partial N$ consists of arcs. We have 
$\alpha^{\prime} \subset B^{+}\left(S^{\prime}\right) \subset B^{+}\left(S^{\Gamma}\right)$. It follows $\partial N \cap \alpha^{\prime}=\emptyset$, since otherwise a sub-disc of $D^{\prime}$ is a compressing disc for $\partial N \cap B^{+}\left(S^{\Gamma}\right)$, which is impossible as $\partial N \cap B^{+}\left(S^{\Gamma}\right)$ is 1-normal by hypothesis. Thus $\partial N \cap \alpha^{\prime}=\emptyset$ and $D^{\prime} \subset N$.

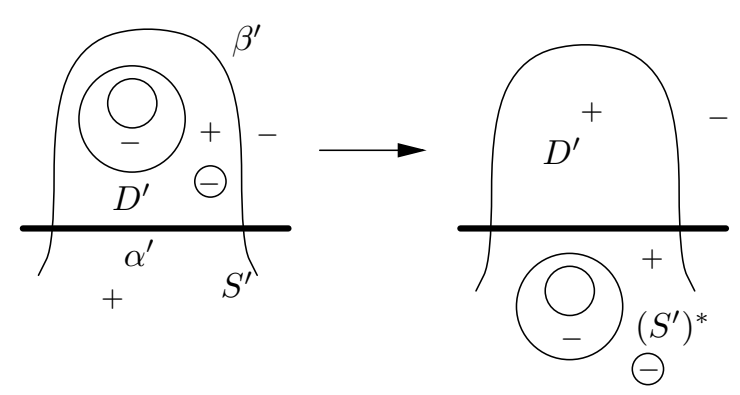

Figure 3: How to produce a bond

By an isotopy with support in $U\left(D^{\prime}\right)$ that is constant on $\beta^{\prime}$, we move $\left(D^{\prime} \cap\right.$ $\left.S^{\prime}\right) \backslash \beta^{\prime}$ to $U\left(D^{\prime}\right) \backslash t$, and obtain from $S^{\prime}$ a surface $\left(S^{\prime}\right)^{*} \subset N$ that has $D^{\prime}$ as upper bond. This is shown in Figure 3, where $B^{+}\left(S^{\prime}\right)$ is indicated by plus signs and $\mathcal{T}^{1}$ is bold. The isotopy moves $\Gamma$ to a system of arcs $\Gamma^{*} \subset N$ and moves $S^{\Gamma}$ to $S^{\Gamma^{*}}$ with $\Gamma^{*} \subset B^{-}\left(S^{\Gamma^{*}}\right)$. Since $\alpha^{\prime} \subset B^{+}\left(S^{\prime}\right)$, there is a homeomorphism $\varphi: B^{-}\left(S^{\prime}\right) \rightarrow B^{-}\left(\left(S^{\prime}\right)^{*}\right)$ that is constant on $\mathcal{T}^{1}$ with $\varphi\left(B^{-}\left(S^{\Gamma}\right)\right)=B^{-}\left(S^{\Gamma^{*}}\right)$. One obtains $S^{\prime}$ by a sequence of elementary reductions along bonds of $S^{\Gamma}$ that are contained in $B^{-}\left(S^{\prime}\right)$. These bonds are carried by $\varphi$ to bonds of $S^{\Gamma^{*}}$. Thus $\left(S^{\prime}\right)^{*}$ is an upper reduction of $S^{\Gamma^{*}}$. Since $\left(S^{\prime}\right)^{*}$ admits an elementary reduction along its upper bond $D^{\prime}$, we obtain a contradiction to the minimality of $\left\|S^{\prime}\right\|$. Thus $S^{\prime}$ is almost 1 -normal.

Lemma 13 Let $\Gamma$ and $S^{\prime}$ be as in the previous lemma, and let $G_{1}, G_{2}$ be two connected components of $\left(S^{\prime}\right)^{\times}$that both split $M$. Then there is no arc in $\left(\mathcal{T}^{1} \backslash \mathcal{T}^{0}\right) \cap B^{+}\left(S^{\prime}\right) \cap N$ joining $G_{1}$ with $G_{2}$.

Proof By the previous lemma, $S^{\prime}$ is almost 1-normal. Recall that one obtains $\left(S^{\prime}\right)^{\times}$up to isotopy mod $\mathcal{T}^{1}$ by splitting $S^{\prime}$ along splitting discs that do not meet $\mathcal{T}^{2}$. Assume that there is an arc $\alpha \subset\left(\mathcal{T}^{1} \backslash \mathcal{T}^{0}\right) \cap B^{+}\left(S^{\prime}\right) \cap N$ joining $G_{1}$ with $G_{2}$. Let $Y$ be the component of $M \backslash\left(G_{1} \cup G_{2}\right)$ that contains $\alpha$.

By hypothesis, $S^{\Gamma}$ is connected. Thus $S^{\prime}$ is connected, and there is an arc $\beta \subset S^{\prime}$ with $\partial \beta=\partial \alpha$. Since $G_{1}, G_{2}$ split $M$, the set $Y$ is the only component of $M \backslash\left(G_{1} \cup G_{2}\right)$ with boundary $G_{1} \cup G_{2}$. Thus there is a component $\beta^{\prime}$ of $\beta \cap Y$ connecting $G_{1}$ with $G_{2}$. There is a splitting disc $D \subset Y$ of $S^{\prime}$ contained in a single tetrahedron with $\beta^{\prime} \cap D \neq \emptyset$. By choosing $D$ innermost, we assume that 
$\beta \cap D$ is a single point in $\partial D$. Since $\partial N$ is pre-normal and $D$ is contained in a single tetrahedron, we can assume by isotopy of $D \bmod \mathcal{T}^{2}$ that $D \cap \partial N=\emptyset$, thus $D \subset N$.

Choose a disc $D^{\prime} \subset U(\alpha \cup \beta) \cap B^{+}\left(S^{\prime}\right)$ so that $D^{\prime} \cap \mathcal{T}^{1}=\alpha$ and $D^{\prime} \cap S^{\prime}=$ $\beta \backslash U(\partial D)$. Then $D^{\prime} \cap \partial N=\emptyset$, since $U(\alpha \cup \beta) \cap \partial N=\emptyset$. We split $S^{\prime}$ along $D$, pull the two components of $\left(S^{\prime} \cap \partial U(D)\right) \backslash D$ along $\left(\partial D^{\prime}\right) \backslash(\alpha \cup \beta)$, and reglue. We obtain a surface $\left(S^{\prime}\right)^{*}$ with $D^{\prime}$ as an upper bond.

Since a small collar of $\partial D$ in $D$ is in $B^{-}\left(S^{\prime}\right)$, there is a homeomorphism $\varphi: B^{-}\left(S^{\prime}\right) \rightarrow B^{-}\left(\left(S^{\prime}\right)^{*}\right)$ that is constant on $\mathcal{T}^{1}$. Set $\Gamma^{*}=\varphi(\Gamma)$. Then $\varphi\left(S^{\Gamma}\right)=$ $S^{\Gamma^{*}}$ with $\Gamma^{*} \subset B^{-}\left(S^{\Gamma^{*}}\right)$. As in the proof of the previous lemma, $\left(S^{\prime}\right)^{*}$ is an upper reduction of $S^{\Gamma^{*}}$, and $\left(S^{\prime}\right)^{*}$ admits an elementary reduction along $D^{\prime}$. This contradiction to the minimality of $\left\|S^{\prime}\right\|$ yields the lemma.

\section{Proof of Theorem 2}

Let $\mathcal{T}$ be a triangulation of $S^{3}$ with a vertex $x_{0} \in \mathcal{T}^{0}$. Let $\Sigma \subset S^{3}$ be a maximal system of disjoint 1 -normal spheres with $\|\Sigma\|<2^{185 t(\mathcal{T})^{2}}$, as given by Construction 1. Construction 2 extends $\Sigma$ to a system $\tilde{\Sigma} \subset S^{3}$ of disjoint 2 -normal spheres that are pairwise non-isotopic $\bmod \mathcal{T}^{2}$, such that

(1) any component of $\tilde{\Sigma}$ has at most one octagon,

(2) any component of $S^{3} \backslash \tilde{\Sigma}$ has at most one boundary component that is not 1-normal,

(3) if the boundary of a component $N$ of $S^{3} \backslash \tilde{\Sigma}$ is 1 -normal, then $N$ does not contain 2 -normal spheres with exactly one octagon, and

(4) $\|\tilde{\Sigma}\|<2^{190 t(\mathcal{T})^{2}}$.

Let $N$ be a component of $S^{3} \backslash \tilde{\Sigma}$ that is not a regular neighbourhood of a vertex of $\mathcal{T}$. Let $S_{0}$ be the component of $\partial N$ with $N \subset B^{-}\left(S_{0}\right)$, and let $S_{1}, \ldots, S_{k}$ be the other components of $\partial N$. Since $\Sigma$ is maximal, any almost 1-normal sphere in $N$ is a tube sum of copies of $S_{0}, S_{1}, \ldots, S_{k}$.

Lemma $14 N \cap \mathcal{T}^{0}=\emptyset$.

Proof If $x \in N \cap \mathcal{T}^{0}$, then the sphere $\partial U(x) \subset N$ is 1 -normal. It is not isotopic $\bmod \mathcal{T}^{1}$ to a component of $\partial N$, since $N \neq U(x)$. This contradicts the maximality of $\Sigma$. 
Lemma 15 If $\partial N$ is 1-normal, then there is an arc in $\mathcal{T}^{1} \cap \bar{N}$ that connects two different components of $\partial N \backslash S_{0}$.

Proof Let $\partial N=S_{0} \cup S_{1} \cup \cdots \cup S_{k}$ be 1-normal. We first consider the case where there is an almost 1 -normal sphere $S \in S_{1} \circ \cdots \circ S_{k}$ in $\bar{N}$ that has a compressing disc $D$, with string $\alpha$ and base $\beta$. We choose $D$ innermost, so that $\alpha \cap S=\partial \alpha$. In particular, $\alpha \cap \partial N=\partial \alpha$. Assume that $\alpha \not \subset \bar{N}$. Since $\partial D \backslash \alpha \subset \bar{N}$, there is an arc $\beta^{\prime} \subset D \cap \partial N$ that connects the endpoints of $\alpha$. The sub-disc $D^{\prime} \subset D$ bounded by $\alpha \cup \beta^{\prime}$ is a compressing disc for the 1 -normal surface $\partial N$, in contradiction to Lemma 10. By consequence, $\alpha \subset \bar{N}$. Assume that $\partial \alpha$ is contained in a single component of $\partial N \backslash S_{0}$, say, in $S_{1}$. By Lemma $10, D$ is not a compressing disc for $S_{1}$, hence $\beta \not \subset S_{1}$. Thus there is a closed line in $S_{1} \backslash \beta$ that separates $\partial \alpha$ on $S_{1}$, but not on $S$. This is impossible as $S$ is a sphere. We conclude that if $S$ has a compressing disc, then there is an $\operatorname{arc} \alpha \subset \mathcal{T}^{1} \cap N$ that connects different components of $\partial N \backslash S_{0}$.

It remains to consider the case where no sphere in $S_{1} \circ \cdots \circ S_{k}$ contained in $\bar{N}$ has a compressing disc. We will show the existence of an almost 2 -normal sphere in $N$ with exactly one octagon, using the technique of thin position. This contradicts property (3) of $\tilde{\Sigma}$ (see the begin of this section), and therefore finishes the proof of the lemma. Let $J: S^{2} \times I \rightarrow B^{-}\left(S_{0}\right)$ be a $\mathcal{T}^{1}$-Morse embedding, such that

(1) $J\left(S^{2} \times 0\right)=S_{0}$,

(2) $J\left(S^{2} \times \frac{1}{2}\right) \in S_{1} \circ \cdots \circ S_{k}$ (or $\left\|J\left(S^{2} \times \frac{1}{2}\right)\right\|=0$, in the case $\left.\partial N=S_{0}\right)$,

(3) $B^{-}\left(J\left(S^{2} \times 1\right)\right) \cap \mathcal{T}^{1}=\emptyset$, and

(4) $\kappa(J)$ is minimal.

Define $S=J\left(S^{2} \times \frac{1}{2}\right)$. Assume that for some $\xi \in I$ there is a pair $D_{1}, D_{2} \subset M$ of nested or independent upper and lower compressing discs for $J_{\xi}=J\left(S^{2} \times \xi\right)$. We show that we can assume $D_{1}, D_{2} \subset B^{-}\left(S_{0}\right)$. Since $S_{0}$ is 1-normal, it has no compressing discs by Lemma 10 . Thus $\left(D_{1} \cup D_{2}\right) \cap S_{0}$ consists of circles. Any such circle bounds a disc in $S_{0} \backslash \mathcal{T}^{1}$ by Lemma 11. By cut-and-paste of $D_{1} \cup D_{2}$, we obtain $D_{1}, D_{2} \subset B^{-}\left(S_{0}\right)$, as claimed. Now, one obtains from $J$ an embedding $J^{\prime}: S^{2} \times I \rightarrow B^{-}\left(S_{0}\right)$ with $\kappa\left(J^{\prime}\right)<\kappa(J)$ by isotopy along $D_{1} \cup D_{2}$, see [16], [22]. The embedding $J^{\prime}$ meets conditions (1) and (3) in the definition of $J$. Since $S \in S_{1} \circ \cdots \circ S_{k}$ has no compressing discs by assumption, $S \cap D_{i}$ consists of circles. Thus $S$ is split equivalent to $J^{\prime}\left(S^{2} \times \frac{1}{2}\right)$. So $J^{\prime}$ meets also condition (2), $J^{\prime}\left(S^{2} \times \frac{1}{2}\right) \in S_{1} \circ \cdots \circ S_{k}$, in contradiction to the choice of $J$. This disproves the existence of $D_{1}, D_{2}$. In conclusion, if $J_{\xi}$ has upper and lower bonds, then it is impermeable. 
Let $\xi_{\max }$ be the greatest critical parameter of $J$ with respect to $\mathcal{T}^{1}$ in the interval $] 0, \frac{1}{2}\left[\right.$. We have $N \cap \mathcal{T}^{0}=\emptyset$ by Lemma 14 . Hence the critical point corresponding to $\xi_{\max }$ is a point of tangency of $J_{\xi_{\max }}$ to some edge of $\mathcal{T}$. By assumption, $S$ has no upper bonds, thus $\|S\|<\left\|J_{\xi_{\max }-\epsilon}\right\|$ for sufficiently small $\epsilon>0$. Let $\xi_{\min } \in I$ be the smallest critical parameter of $J$ with respect to $\mathcal{T}^{1}$. By Lemma 10, $S_{0}$ has no bonds, thus $\left\|S_{0}\right\|<\left\|J_{\xi_{\text {min }}+\epsilon}\right\|$. Therefore there are consecutive critical parameters $\left.\xi_{1}, \xi_{2} \in\right] 0, \frac{1}{2}[$ such that

$$
\left\|J_{\xi_{1}-\epsilon}\right\|<\left\|J_{\xi_{1}+\epsilon}\right\|>\left\|J_{\xi_{2}+\epsilon}\right\| \text {. }
$$

Thus $J_{\xi_{1}+\epsilon}$ has both upper and lower bonds, and is therefore impermeable by the preceding paragraph. One component of $J_{\xi_{1}+\epsilon}^{\times}$is a 2-normal sphere in $N$ with exactly one octagon, by Proposition 3 . The existence of that $2-$ normal sphere is a contradiction to the properties of $\tilde{\Sigma}$, which proves the lemma.

We show that some tube sum $S \in S_{1} \circ \cdots \circ S_{k}$ is isotopic to $S_{0}$ such that $\|\cdot\|$ is monotone under the isotopy. We consider three cases. In the first case, let $\partial N$ be $1-$ normal.

Lemma 16 If $\partial N$ is 1-normal, then there is a sphere $S \in S_{1} \circ \cdots \circ S_{k}$ in $N$ with an upper reduction $S^{\prime} \subset N$ so that there is a $\mathcal{T}^{1}$-Morse embedding $J: S^{2} \times I \rightarrow S^{3}$ with $J\left(S^{2} \times I\right)=B^{+}\left(S^{\prime}\right) \cap B^{-}\left(S_{0}\right)$ and $c\left(J, \mathcal{T}^{1}\right)=0$.

Proof By Lemma 15, there is an arc $\alpha \subset \mathcal{T}^{1} \cap N$ that connects two components of $\partial N \backslash S_{0}$, say, $S_{1}$ with $S_{2}$. By Lemma $14, \alpha$ is contained in an edge of $\mathcal{T}$. By Lemma 10, the 1-normal surfaces $S_{1}, \ldots, S_{k}$ have no lower compressing discs. Let $\Gamma \subset N$ be a system of $k-1$ arcs, such that the tube sum $S$ of $S_{1}, \ldots, S_{k}$ along $\Gamma$ is a sphere and an upper reduction $S^{\prime} \subset N$ of $S$ minimizes $\left\|S^{\prime}\right\|$. We have $\left\|S^{\prime}\right\|<\|S\|$, since it is possible to choose $\Gamma$ so that $S$ has an upper bond with string $\alpha$. Since $\Gamma \subset B^{-}(S)$ and by Lemma 12, $S^{\prime}$ is almost 1 -normal.

By the maximality of $\Sigma$, it follows $S^{\prime} \in n_{0} S_{0} \circ \cdots \circ n_{k} S_{k}$ with non-negative integers $n_{0}, n_{1}, \ldots, n_{k}$. Moreover, $n_{i} \leq 2$ for $i=0, \ldots, k$ by Lemma 13 . Since $S$ separates $S_{0}$ from $S_{1}, \ldots, S_{k}$, so does $S^{\prime}$. Thus any path connecting $S_{0}$ with $S_{j}$ for some $j \in\{1, \ldots, k\}$ intersects $S^{\prime}$ in an odd number of points. So alternatively $n_{0} \in\{0,2\}$ and $n_{i}=1$ for all $i \in\{1, \ldots, k\}$, or $n_{0}=1$ and $n_{i} \in\{0,2\}$ for all $i \in\{1, \ldots, k\}$. Since $\left\|S^{\prime}\right\|<\left\|S^{*}\right\|$, it follows $n_{0}=1$ and $n_{i}=0$ for $i \in\{1, \ldots, k\}$, thus $\left(S^{\prime}\right)^{\times}=S_{0}$. The existence of a $\mathcal{T}^{1}$-Morse embedding $J$ with the claimed properties follows then by Corollary 1 .

The second case is that $S_{0}$ is 1 -normal, and exactly one of $S_{1}, \ldots, S_{k}$ contains exactly one octagon, say, $S_{1}$. The octagon gives rise to an upper bond $D$ of $S_{1}$ 
contained in a single tetrahedron. Since $\partial N \backslash S_{1}$ is 1 -normal, $D \subset N$. Thus an elementary reduction of $S_{1}$ along $D$ transforms $S_{1}$ to a sphere $F \subset N$. Since $S_{1}$ is impermeable by Proposition $4, F$ has no lower compressing disc (such a disc would give rise to a lower compressing disc for $S_{1}$ that is independent from $D)$.

Lemma 17 If $\partial N \backslash S_{0}$ is not 1-normal, then there is a sphere $S \in S_{1} \circ \cdots \circ S_{k}$ in $N$ with an upper reduction $S^{\prime} \subset N$ so that there is a $\mathcal{T}^{1}$-Morse embedding $J: S^{2} \times I \rightarrow S^{3}$ with $J\left(S^{2} \times I\right)=B^{+}\left(S^{\prime}\right) \cap B^{-}\left(S_{0}\right)$ and $c\left(J, \mathcal{T}^{1}\right)=0$.

Proof We apply the Lemma 12 to $F, S_{2}, \ldots, S_{k}$, and together with the elementary reduction along $D$ we obtain a sphere $S \in S_{1} \circ S_{2} \circ \cdots \circ S_{k}$ with an almost 1-normal upper reduction $S^{\prime} \subset N$. One concludes $\left(S^{\prime}\right)^{\times}=S_{0}$ and the existence of $J$ as in the proof of the previous lemma.

We come to the third and last case, namely $S_{0}$ has exactly one octagon and $\partial N \backslash S_{0}$ is 1 -normal. The octagon gives rise to a lower bond $D$ of $S_{0}$, that is contained in $N$ since $\partial N \backslash S_{0}$ is 1 -normal. Thus an elementary reduction of $S_{0}$ along $D$ yields a sphere $F \subset N$. Since $S_{0}$ is impermeable by Proposition 4, $F$ has no upper compressing disc, similar to the previous case.

Lemma 18 If $S_{0}$ is not 1-normal, then there is a lower reduction $S^{\prime} \in S_{1}$ 。 $\cdots \circ S_{k}$ of $S_{0}$, with $S^{\prime} \subset N$.

Proof We apply Lemma 12 with $\Gamma=\emptyset$ to lower reductions of $F$, which is possible by symmetry. Thus, together with the elementary reduction along $D$, there is a lower reduction $S^{\prime} \in n_{0} S_{0} \circ \cdots \circ n_{k} S_{k}$ of $S_{0}$, and $n_{0}, \ldots, n_{k} \leq 2$ by Lemma 13. Since $S^{\prime} \subset B^{-}(F)$ and $S_{0} \subset B^{+}(F)$, it follows $n_{0}=0$. Since $S^{\prime}$ separates $\partial N \cap B^{+}(F)$ from $\partial N \cap B^{-}(F)$, it follows $n_{1}, \ldots, n_{k}$ odd, thus $n_{1}=\cdots=n_{k}=1$.

We are now ready to construct the $\mathcal{T}^{1}$-Morse embedding $H: S^{2} \times I \rightarrow S^{3}$ with $c\left(H, \mathcal{T}^{1}\right)$ bounded in terms of $t(\mathcal{T})$, thus to finish the proof of Theorems 1 and 2. Let $x_{0} \in \mathcal{T}^{0}$ be the vertex involved in the definition of $B^{+}(\cdot)$. We construct $H$ inductively as follows.

Choose $\left.\xi_{1} \in\right] 0,1\left[\right.$ and choose $H \mid\left[0, \xi_{1}\right]$ so that $H_{0} \cap \mathcal{T}^{2}=\emptyset, H_{\xi_{1}}=\partial U\left(x_{0}\right) \subset \tilde{\Sigma}$, and $x_{0}$ is the only critical point of $H \mid\left[0, \xi_{1}\right]$.

For $i \geq 1$, let $H \mid\left[0, \xi_{i}\right]$ be already constructed. Our induction hypothesis is that $H_{\xi_{i}} \in S_{0} \circ S^{*}$ for some component $S_{0}$ of $\tilde{\Sigma}$, and moreover for any choice of $S_{0}$ we have $H_{\xi_{i}} \subset B^{+}\left(S_{0}\right)$. Choose $\left.\xi_{i+1} \in\right] \xi_{i}, 1[$. 
Assume that $S_{0}$ is not of the form $S_{0}=\partial U(x)$ for a vertex $x \in \mathcal{T}^{0} \backslash\left\{x_{0}\right\}$. Then, let $N_{i}$ be the component of $S^{3} \backslash \tilde{\Sigma}$ with $N_{i} \subset B^{-}\left(S_{0}\right)$ and $\partial N_{i}=S_{0} \cup S_{1} \cup \cdots \cup S_{k}$ for $S_{1}, \ldots, S_{k} \subset \tilde{\Sigma}$. If $S_{0}$ is 1 -normal, then let $S \in S_{1} \circ \cdots \circ S_{k}, S^{\prime}$ and $J$ be as in Lemmas 16 and 17. Then, we extend $H \mid\left[0, \xi_{i}\right]$ to $H \mid\left[0, \xi_{i+1}\right]$ induced by the embedding $J$, relating $S_{0}$ with $S^{\prime}$, and by the inverses of the elementary upper reductions, relating $S^{\prime}$ with $S$. If $S_{0}$ is not 1 -normal, then let $S \in S_{1} \circ \cdots \circ S_{k}$ be as in Lemma 18. We extend $H \mid\left[0, \xi_{i}\right]$ to $H \mid\left[0, \xi_{i+1}\right]$ along the elementary lower reductions, relating $S_{0}$ with $S$. In either case, $H_{\xi_{i+1}} \in S_{1} \circ \cdots \circ S_{k} \circ S^{*}$. The critical points of $H \mid\left[\xi_{i}, \xi_{i+1}\right]$ are contained in $N_{i}$, given by elementary reductions. Thus the number of these critical points is $\leq \frac{1}{2} \max \left\{\left\|S_{0}\right\|,\|S\|\right\} \leq$ $\frac{1}{2}\|\tilde{\Sigma}\|<2^{190 t(\mathcal{T})^{2}}$, by Construction 2. Since $H_{\xi_{i+1}} \subset B^{+}\left(S_{m}\right)$ for any $m=$ $1, \ldots, k$, we can proceed with our induction.

After at most \# $(\tilde{\Sigma})$ steps, we have $H_{\xi_{i}}^{\times}=\partial U\left(\mathcal{T}^{0} \backslash\left\{x_{0}\right\}\right)$. Then, choose $H \mid\left[\xi_{i}, 1\right]$ so that $H_{1} \cap \mathcal{T}^{2}=\emptyset$ and the set of its critical points is $\mathcal{T}^{0} \backslash\left\{x_{0}\right\}$. By Proposition 2 holds $\#(\tilde{\Sigma}) \leq 10 t(\mathcal{T})$. Thus finally

$$
c\left(H, \mathcal{T}^{1}\right)<\#\left(\mathcal{T}^{0}\right)+10 t(\mathcal{T}) \cdot 2^{190 t(\mathcal{T})^{2}}<2^{196 t(\mathcal{T})^{2}} .
$$

\section{Proof of Propositions 3 and 4}

Let $M$ be a closed connected 3 -manifold with a triangulation $\mathcal{T}$. We prove Proposition 3, that states that any impermeable surface in $M$ is isotopic mod $\mathcal{T}^{1}$ to an almost $2-$ normal surface with exactly one octagon. The proof consists of the following three lemmas.

Lemma 19 Any impermeable surface in $M$ is almost 2-normal, up to isotopy $\bmod \mathcal{T}^{1}$.

Proof We give here just an outline. A complete proof can be found in [16]. Let $S \subset M$ be an impermeable surface. By definition, it has upper and lower bonds with strings $\alpha_{1}, \alpha_{2}$. By isotopies $\bmod \mathcal{T}^{1}$, one obtains from $S$ two surfaces $S_{1}, S_{2} \subset M$, such that $S_{i}$ has a return $\beta_{i} \subset \mathcal{T}^{2}$ with $\partial \beta_{i}=\partial \alpha_{i}$, for $i \in\{1,2\}$. A surface that has both upper and lower returns admits an independent pair of upper and lower compressing discs, thus is not impermeable. By consequence, under the isotopy mod $\mathcal{T}^{1}$ that relates $S_{1}$ and $S_{2}$ occurs a surface $S^{\prime}$ that has no returns at all, thus is almost $k$-normal for some natural number $k$.

If there is a boundary component $\zeta$ of a component of $S^{\prime} \backslash \mathcal{T}^{2}$ and an edge $e$ of $\mathcal{T}$ with $\#(\zeta \cap e)>2$, then there is an independent pair of upper and lower compressing discs. Thus $k=2$. 
Lemma 20 Let $S \subset M$ be an almost 2-normal impermeable surface. Then $S$ contains at most one octagon.

Proof Two octagons in different tetrahedra of $\mathcal{T}$ give rise to a pair of independent upper and lower compressing discs for $S$. Two octagons in one tetrahedron of $\mathcal{T}$ give rise to a pair of nested upper and lower compressing discs for $S$. Both is a contradiction to the impermeability of $S$.

Lemma 21 Let $S \subset M$ be an almost 2-normal impermeable surface. Then $S$ contains at least one octagon.

Proof By hypothesis, $S$ has both upper and lower bonds. Assume that $S$ does not contain octagons, i.e., it is almost 1 -normal. We will obtain a contradiction to the impermeability of $S$ by showing that $S$ has a pair of independent or nested compressing discs.

According to Lemma 10, we can assume that $S$ has a compressing disc $D_{1}$ with string $\alpha_{1}$ that is contained in a single closed tetrahedron $t_{1}$. Choose $D_{1}$ innermost, i.e., $\alpha_{1} \cap S=\partial \alpha_{1}$. Without assumption, let $D_{1}$ be upper. Since $S$ has no octagon by assumption, $\alpha_{1}$ connects two different components $\zeta_{1}, \eta_{1}$ of $S \cap \partial t_{1}$. Let $D$ be a lower bond of $S$. Choose $S, D_{1}$ and $D$ so that, in addition, $\#\left(D \cap \mathcal{T}^{2}\right)$ is minimal.

Let $C$ be the closure of an innermost component of $D \backslash \mathcal{T}^{2}$, which is a disc. There is a closed tetrahedron $t_{2}$ of $\mathcal{T}$ and a closed 2-simplex $\sigma_{2} \subset \partial t_{2}$ of $\mathcal{T}$ such that $\partial C \cap \partial t_{2}$ is a single component $\gamma \subset \sigma_{2}$. We have to consider three cases.

(1) Let $\gamma$ be a circle, thus $\partial C=\gamma$. There is a disc $D^{\prime} \subset \sigma_{2}$ with $\partial D^{\prime}=\gamma$ and a ball $B \subset t_{2}$ with $\partial B=C \cup D^{\prime}$. We move $S \cup D$ away from $B$ by an isotopy mod $\mathcal{T}^{1}$ with support in $U(B)$, and obtain a surface $S^{*}$ with a lower bond $D^{*}$. As $D$ is a bond, $S \cap D^{\prime}$ consists of circles. Therefore the normal arcs of $S \cap \mathcal{T}^{2}$ are not changed under the isotopy, and the isotopy does not introduce returns, thus $S^{*}$ is almost 1 -normal. Since $\xi_{1} \cap D^{\prime}=\eta_{1} \cap D^{\prime}=\emptyset$ and $C \cap S=\emptyset$, it follows $B \cap \partial D_{1}=\emptyset$. Thus $D_{1}$ is an upper compressing disc for $S^{*}$, and $\#\left(D^{*} \cap \mathcal{T}^{2}\right)<\#\left(D \cap \mathcal{T}^{2}\right)$ in contradiction to our choice.

(2) Let $\gamma$ be an arc with endpoints in a single component $c$ of $S \cap \sigma$. By an isotopy mod $\mathcal{T}^{1}$ with support in $U(C)$ that moves $C$ into $U(C) \backslash t_{2}$, we obtain from $S$ and $D$ a surface $S^{*}$ with a lower bond $D^{*}$. Since $D$ is a bond, the isotopy does not introduce returns, thus $S^{*}$ is almost 1 -normal. 
One component of $S^{*} \cap t_{1}$ is isotopic mod $\mathcal{T}^{2}$ to the component of $S \cap t_{1}$ that contains $\partial D_{1} \cap S$. Thus up to isotopy $\bmod \mathcal{T}^{2}, D_{1}$ is an upper compressing disc for $S^{*}$, and $\#\left(D^{*} \cap \mathcal{T}^{2}\right)<\#\left(D \cap \mathcal{T}^{2}\right)$ in contradiction to our choice.

(3) Let $\gamma$ be an arc with endpoints in two different components $c_{1}, c_{2}$ of $S \cap \sigma$. Assume that, say, $c_{1}$ is a circle. By an isotopy mod $\mathcal{T}^{1}$ with support in $U(C)$ that moves $C$ into $U(C) \backslash t_{2}$, we obtain from $S$ and $D$ a surface $S^{*}$ with a lower bond $D^{*}$. Since $D$ is a bond, the isotopy does not introduce returns, thus $S^{*}$ is almost $1-$ normal. There is a disc $D^{\prime} \subset \sigma$ with $\partial D^{\prime}=c_{1}$. Let $K$ be the component of $S \cap t_{1}$ that contains $\partial D_{1} \cap S$. One component of $S^{*} \cap t_{1}$ is isotopic mod $\mathcal{T}^{2}$ either to $K$ or, if $\partial D^{\prime} \cap \partial K \neq \emptyset$, to $K \cup D^{\prime}$. In either case, $D_{1}$ is an upper compressing disc for $S^{*}$, up to isotopy $\bmod \mathcal{T}^{2}$. But $\#\left(D^{*} \cap \mathcal{T}^{2}\right)<\#\left(D \cap \mathcal{T}^{2}\right)$ in contradiction to our choice. Thus, $c_{1}$ and $c_{2}$ are normal arcs.

Since $S$ is almost $1-$ normal, $c_{1}, c_{2}$ are contained in different components $\zeta_{2}, \eta_{2}$ of $S \cap \partial t_{2}$. Since $D$ is a lower bond, $\partial\left(C \cap D_{1}\right) \subset \partial C \cap S$. There is a sub-arc $\alpha_{2}$ of an edge of $t_{2}$ and a disc $D^{\prime} \subset \sigma$ with $\partial D^{\prime} \subset \alpha_{2} \cup \gamma \cup \zeta_{2} \cup \eta_{2}$ and $\alpha_{2} \cap S=\partial \alpha_{2}$. The disc $D_{2}=C \cup D^{\prime} \subset t_{2}$ is a lower compressing disc for $S$ with string $\alpha_{2}$, and $\partial\left(D_{1} \cap D_{2}\right) \subset \partial D_{2} \cap S$. At least one component of $\partial t_{1} \backslash\left(\zeta_{1} \cup \eta_{1}\right)$ is a disc that is disjoint to $D_{2}$. Let $D_{m}$ be the closure of a copy of such a disc in the interior of $t_{1}$, with $\partial D_{m} \subset S$. By construction, $D_{1} \cap D_{m}=\partial D_{1} \cap \partial D_{m}$ is a single point and $D_{2} \cap D_{m}=\emptyset$. Thus by Lemma $9, S$ has a pair of independent or nested upper and lower compressing discs and is therefore not impermeable.

Proof of Proposition 4 Let $S \subset M$ be a connected 2-normal surface that splits $M$, and assume that exactly one component $O$ of $S \backslash \mathcal{T}^{2}$ is an octagon. The octagon gives rise to upper and lower bonds of $S$.

Let $D_{1}, D_{2}$ be any upper and lower compressing discs for $S$. We have to show that $D_{1}$ and $D_{2}$ are neither impermeable nor nested. It suffices to show that $\partial D_{1} \cap \partial D_{2} \not \subset \mathcal{T}^{1}$. To obtain a contradiction, assume that $\partial D_{1} \cap \partial D_{2} \subset \mathcal{T}^{1}$. Choose $D_{1}, D_{2}$ so that $\#\left(\partial D_{1} \backslash \mathcal{T}^{2}\right)+\#\left(\partial D_{2} \backslash \mathcal{T}^{2}\right)$ is minimal.

Let $t$ be a tetrahedron of $\mathcal{T}$ with a closed 2-simplex $\sigma \subset \partial t$, and let $\beta$ be a component of $\partial D_{1} \cap t$ (resp. $\partial D_{2} \cap t$ ) such that $\partial \beta$ is contained in a single component of $S \cap \sigma$. Since $S$ is 2 -normal, there is a disc $D \subset S \cap t$ and an arc $\gamma \subset S \cap \sigma$ with $\partial D=\beta \cup \gamma$. By choosing $\beta$ innermost in $D$, we can assume that $D \cap\left(\partial D_{1} \cup \partial D_{2}\right)=\beta$. An isotopy of $\left(D_{1}, \partial D_{1}\right)\left(\operatorname{resp} .\left(D_{2}, \partial D_{2}\right)\right)$ in $(M, S)$ with support in $U(D)$ that moves $\beta$ to $U(D) \backslash t$ reduces \# $\left(\partial D_{1} \backslash \mathcal{T}^{2}\right)$ (resp. 
$\left.\#\left(\partial D_{2} \backslash \mathcal{T}^{2}\right)\right)$, leaving $\partial D_{1} \cap \partial D_{2}$ unchanged. This is a contradiction to the minimality of $D_{1}, D_{2}$.

For $i=1,2$, there are $\operatorname{arcs} \beta_{i} \subset \partial D_{i} \backslash \mathcal{T}^{1}$ and $\gamma_{i} \subset D_{i} \cap \mathcal{T}^{2}$ such that $\beta_{i} \cup \gamma_{i}$ bounds a component of $D_{i} \backslash \mathcal{T}^{2}$, by an innermost arc argument. Let $t_{i}$ be the tetrahedron of $\mathcal{T}$ that contains $\beta_{i}$, and let $\sigma_{i} \subset \partial t_{i}$ be the close 2 -simplex that contains $\gamma_{i}$. We have seen above that $\partial \beta_{i}$ is not contained in a single component of $S \cap \sigma_{i}$. Since $S$ is 2 -normal, i.e., has no tubes, it follows that $\beta_{i} \subset O$. Since collars of $\beta_{1}$ in $D_{1}$ and of $\beta_{2}$ in $D_{2}$ are in different components of $t \backslash O$, it follows $\beta_{1} \cap \beta_{2} \neq \emptyset$. Thus $\partial D_{1} \cap \partial D_{2} \not \subset \mathcal{T}^{1}$, which yields Proposition 4 .

\section{References}

[1] S Armentrout, Knots and shellable cell partitionings of $S^{3}$, Illinois J. Math. 38 (1994) 347-365

[2] G Burde, H Zieschang, Knots, De Gruyter studies in mathematics 5, De Gruyter (1985)

[3] R Ehrenborg, M Hachimori, Non-constructible complexes and the bridge index, preprint

[4] Furch, Zur Grundlegung der kombinatorischen Topologie, Abh. Math. Sem. Hamb. Univ. 3 (1924) 60-88

[5] D Gabai, Foliations and the topology of 3-manifolds III, J. Differ. Geom. 26 (1987) 445-503

[6] R E Goodrick, Non-simplicially collapsible triangulations of $I^{n}$, Proc. Camb. Phil. Soc. 64 (1968) 31-36

[7] M Hachimori, G Ziegler, Decompositions of simplicial balls and spheres with knots consisting of few edges, Math. Z. 235 (2000) 159-171

[8] W Haken, Über das Homöomorphieproblem der 3-Mannigfaltigkeiten I, Math. Z. 80 (1962) 89-120

[9] W Haken, Some results on surfaces in 3-manifolds, from: "Studies in Modern Topology", (P Hilton, editor) Math. Assoc. Amer. Stud. Math. 5, Prentice Hall (1968) 39-98

[10] J Hass, L C Lagarias, The number of Reidemeister moves needed for unknotting, J. Amer. Math. Soc. 14 (2001) 399-428

[11] Hemion, The Classification of Knots and 3-Dimensional Spaces, Oxford University Press (1992)

[12] S King, The size of triangulations supporting a given link, preliminary version, arxiv:math.GT/0007032:v2

[13] S King, How to make a triangulation of $S^{3}$ polytopal, preprint (2000) 
[14] H Kneser, Geschlossene Flächen in dreidimensionalen Mannigfaltigkeiten, Jahresber. Deut. Math. Ver. 38 (1929) 248-260

[15] W B R Lickorish, Unshellable triangulations of spheres, European J. Combinatorics, 12 (1991) 527-530

[16] S V Matveev, An algorithm for the recognition of 3-spheres (according to Thompson), Mat. sb. 186 (1995) 69-84, English translation in Sb. Math. 186 (1995)

[17] S V Matveev, On the recognition problem for Haken 3-manifolds, Suppl. Rend. Circ. Mat. Palermo. 49 (1997) 131-148

[18] A Mijatović, Simplifying triangulations of $S^{3}$, preprint (2000)

[19] J H Rubinstein, Polyhedral minimal surfaces, Heegaard splittings and decision problems for 3-dimensional manifolds, from: "Geometric Topology (Athens, GA. 1993)", Stud. Adv. Math. 2.1, Amer. Math. Soc. \& Intl. Press (1997) 1-20

[20] M Scharlemann, A Thompson, Thin position for 3-manifolds. from: "Geometric Topology", AMS Contemporary Math. 164 (1992) 231-238

[21] A Schrijver, Theory of linear and integer programming, Wiley-Interscience, Chichester (1986)

[22] A Thompson, Thin position and the recognition problem for $S^{3}$, Mathematical Research Letters 1 (1994) 613-630

[23] A Thompson, Algorithmic recognition of 3-manifolds, Bull. Am. Math. Soc. 35 (1998) 57-66 